\title{
The Tucson-Melbourne three-nucleon force in the automatized partial-wave decomposition
}

\author{
R. Skibiński ${ }^{1}$,a, J. Golak ${ }^{1}$, K. Topolnicki ${ }^{1}$, H. Witała ${ }^{1}$, H. Kamada ${ }^{2}$, W. Glöckle ${ }^{3}$, and A. Nogga ${ }^{4}$ \\ 1 M. Smoluchowski Institute of Physics, Jagiellonian University, PL-30059 Kraków, Poland \\ 2 Department of Physics, Faculty of Engineering, Kyushu Institute of Technology, Kitakyushu 804-8550, Japan \\ 3 Institut für Theoretische Physik II, Ruhr-Universität Bochum, D-44780 Bochum, Germany \\ 4 Forschungszentrum Jülich, Institut für Kernphysik, Institute for Advanced Simulation and Jülich Center for Hadron Physics, \\ D-52425 Jülich, Germany
}

Received: 18 January 2011 / Revised: 23 February 2011

Published online: 5 April 2011

(C) The Author(s) 2011. This article is published with open access at Springerlink.com

Communicated by M.C. Birse

\begin{abstract}
A recently developed procedure for a partial-wave decomposition of a three-nucleon force is applied to the $\pi-\pi, \pi-\rho$ and $\rho-\rho$ components of the Tucson-Melbourne three-nucleon potential. The resulting matrix elements for the $\pi-\pi$ and $\pi-\rho$ components are compared with the values obtained using the standard approach to the partial-wave decomposition, in which the $\pi$ - $\rho$ expressions for the matrix elements are also derived and presented. Several numerical tests and results for the triton binding energy and the correlation function prove the reliability and efficiency of the new method.
\end{abstract}

\section{Introduction}

The Tucson-Melbourne (TM) three-nucleon force (3NF) $[1-4]$ is an important model of the three-nucleon $(3 \mathrm{~N})$ interaction. It consists of three parts stemming from exchanges of $\pi-\pi, \pi-\rho$ and $\rho-\rho$ mesons. The main ingredient of the TM force, the meson-nucleon scattering amplitude with the off-shell mesons, was derived using the current algebra techniques. This was done in [1] and improved in [3] for the $\pi-\pi$ part. The $\pi-\rho$ and $\rho-\rho$ contributions were derived in $[2,4]$. In [5] the structure of the $\pi-\pi$ part of the TM $3 \mathrm{NF}$ was revisited to achieve a consistency with the chiral symmetry and the modified force is known as the $\mathrm{TM}^{\prime}$ model.

The effects of all terms on the triton binding energy were studied in [6]. It turned out that the $\pi$ - $\rho$ force acts repulsively for the ${ }^{3} \mathrm{H}$ contrarily to the $\pi-\pi$ interaction and combining them leads, for the most of the considered NN potentials, to the ${ }^{3} \mathrm{H}$ binding energy close to the experimental value. The $\rho-\rho$ force has only a small influence on the triton binding energy. A similar behaviour was observed for scattering observables in the three-nucleon system [7]: the largest effects came from the dominant $\pi-\pi$ part and the influence of the $\pi-\rho$ part was smaller and in the opposite direction. The $\rho-\rho$ contribution proved to

\footnotetext{
a e-mail: roman.skibinski@uj.edu.pl
}

be much smaller and practically negligible. However, the results of refs. $[6,7]$ were based on partial waves restricted to the total angular momenta in the two-nucleon subsystem $j \leq 2$. Thus conclusions of [7] are valid only in a low-energy domain of the three-nucleon continuum. For higher energies, where more partial waves are required to achieve convergence, only the $\pi-\pi$ part of the TM force was used (see, e.g., [8-10]). While the inclusion of this main component of the TM $3 \mathrm{NF}$ improves the description of many scattering observables, some serious discrepancies with data remain and they become larger at higher energies. One of the possible explanations for this disagreement is a lack of shorter-range parts of the $3 \mathrm{NF}$ in those calculations, what calls for a reliable and fast method to obtain matrix elements for all components of the TM force in higher partial waves.

Recently, we have proposed a novel, automatized way to perform a partial-wave decomposition of any two- and three-nucleon potential [11]. This approach makes use of a software for symbolic calculations to generate the part of the code which is specific for a considered force model. More precisely, in this way we calculate exactly the isospin- and spin-momentum parts of the nuclear interactions and generate a corresponding FORTRAN (or C) code. That momentum-dependent output forms an integrand for further five-dimensional numerical integrations. 
In this paper we present the results of applying that new scheme to the original TM $3 \mathrm{~N}$ force. They confirm the feasibility and efficiency of our method and its numerical implementation. The existence of such a reliable procedure is especially important in view of available and forthcoming results from the chiral perturbation theory $(\chi \mathrm{PT})[12]$ for $3 \mathrm{~N}$ forces at higher orders of the chiral expansion. A big number of different momentum-spinisospin structures contained in those interactions requires a safe and automatized method to perform partial-wave decompositions, which is guaranteed by our method. Furthermore, our scheme avoids the application of partialwave-decomposed permutation operators when dealing with products of $3 \mathrm{NFs}$ and permutation operators as they are often required, e.g., in 3N Faddeev equations. Such an application is numerically demanding because it requires a huge number of partial waves. Thus, again an efficient, fast and precise method is needed.

Our novel scheme of an automatized partial-wave decomposition (aPWD) is described in sect. 2. Results and additional tests for our numerical realization are presented in sect. 3 and conclusions are given in sect. 4 . The standard PWD of the $\pi-\rho$ component of the TM $3 \mathrm{NF}$ is given in the appendix.

\section{Automatized partial-wave decomposition}

The $3 \mathrm{NF}, V_{123}$, is an indispensable ingredient in a theoretical description of the few-body systems. It can be always written as a sum of three terms

$$
V_{123}=V^{(1)}+V^{(2)}+V^{(3)},
$$

where each $V^{(i)}$ is symmetrical under the exchange of nucleons $j$ and $k(i, j, k=1,2,3, i \neq j \neq k)$. Such a splitting in the case of the $\pi-\pi$ exchange TM 3NF corresponds to the possible choices of the nucleon undergoing off-shell $\pi \mathrm{N}$ scattering.

The 3NF typically enters the dynamical equations via its part $V^{(1)}$. In the case of the three-nucleon bound state, the Faddeev component $\psi$ fulfils the following equation [13]:

$$
\psi=G_{0} t P \psi+\left(1+G_{0} t\right) G_{0} V^{(1)}(1+P) \psi,
$$

where $G_{0}$ is the free $3 \mathrm{~N}$ propagator and $t$ is the two-body $t$ operator generated from a given nucleon-nucleon $(\mathrm{NN})$ potential through the Lippmann-Schwinger equation. The permutation operator $P \equiv P_{12} P_{23}+P_{13} P_{23}$ is given in terms of the transpositions $P_{i j}$, which interchange particles $i$ and $j$. The full bound-state wave function $\Psi$ is then obtained as $\Psi=(1+P) \psi$.

Transition amplitudes for the elastic nucleon-deuteron scattering, $U$, and for the breakup reaction, $U_{0}$, are given as $[14]$

$$
\begin{aligned}
U & =P G_{0}^{-1} \Phi+P T+V^{(1)}(1+P) \Phi+V^{(1)}(1+P) G_{0} T \\
U_{0} & =(1+P) T
\end{aligned}
$$

where the auxiliary state $T$ fulfils the $3 \mathrm{~N}$ Faddeev equation

$$
\begin{aligned}
T= & t P \Phi+\left(1+t G_{0}\right) V^{(1)}(1+P) \Phi+t P G_{0} T \\
& +\left(1+t G_{0}\right) V^{(1)}(1+P) G_{0} T,
\end{aligned}
$$

with $\Phi$ being the initial state composed of the deuteron wave function and a momentum eigenstate of the projectile nucleon.

Equations (2) and (4) are solved $[14,15]$ in the momentum space using $3 \mathrm{~N}$ partial-wave states $|p, q, \alpha\rangle$ in the jJ-coupling $[16,15]$

$$
|p, q, \alpha\rangle \equiv\left|p q(l s) j\left(\lambda \frac{1}{2}\right) I(j I) J M_{J}\right\rangle\left|\left(t \frac{1}{2}\right) T M_{T}\right\rangle
$$

where $p$ and $q$ are magnitudes of the standard Jacobi momenta and $\alpha$ denotes a set of discrete quantum numbers arising in the following way: the spin $s$ of the subsystem composed from nucleons 2 and 3 is coupled with their orbital angular momentum $l$ to the total angular momentum $j$. The spin $\frac{1}{2}$ of the spectator particle 1 couples with its relative orbital angular momentum $\lambda$ to the total angular momentum of nucleon $1, I$. Finally, $j$ and $I$ are coupled to the total $3 \mathrm{~N}$ angular momentum $J$ with the projection $M_{J}$. For the isospin part, the total isospin $t$ of the (23) subsystem is coupled with the isospin $\frac{1}{2}$ of the spectator nucleon to the total $3 \mathrm{~N}$ isospin $T$ with the projection $M_{T}$.

Any three-nucleon force enters eqs. (2)-(4) in the form of $V^{(1)}(1+P)$. Therefore a partial-wave decomposition of $V^{(1)}$ as well as $V^{(1)} P$ has to be performed. The standard approach to perform a partial-wave decomposition of $V^{(1)}$ [17] is very tedious, even with improvements suggested in [18], since each momentum-spin-isospin structure, which occurs in a $3 \mathrm{NF}$, has to be treated separately. In the case when a $3 \mathrm{NF}$ consists of a big number of such structures, like chiral 3NFs at higher orders of the chiral expansion, the traditional approach to a partial-wave decomposition is very inefficient and extremely time consuming. In addition, the application of the permutation operator, when calculating $V^{(1)} P$, causes an additional numerical problem, which originates from a slow convergence of the $V^{(1)} P$ matrix elements with respect to the number of intermediate states $\left|\alpha^{\prime \prime}\right\rangle$ :

$$
\begin{aligned}
& \left\langle p, q, \alpha\left|V^{(1)} P\right| p^{\prime}, q^{\prime}, \alpha^{\prime}\right\rangle= \\
& \quad \int \mathrm{d} p^{\prime \prime} p^{\prime \prime 2} \int \mathrm{d} q^{\prime \prime} q^{\prime \prime 2} \sum_{\alpha^{\prime \prime}}\left\langle p, q, \alpha\left|V^{(1)}\right| p^{\prime \prime}, q^{\prime \prime}, \alpha^{\prime \prime}\right\rangle \\
& \quad \times\left\langle p^{\prime \prime}, q^{\prime \prime}, \alpha^{\prime \prime}|P| p^{\prime}, q^{\prime}, \alpha^{\prime}\right\rangle .
\end{aligned}
$$

In order to calculate precisely these matrix elements, a big number of intermediate states $\left|\alpha^{\prime \prime}\right\rangle$ is required, and, thus, one is forced to calculate the matrix elements of the $V^{(1)}$ operator for a much bigger set of $\alpha^{\prime \prime}$ states than actually needed in order to get converged solutions of the Faddeev equations.

In our new approach, called, in the following, automatized partial-wave decomposition (aPWD), to get matrix elements of $V^{(1)}$ and $V^{(1)} P$, that drawback is removed 
because matrix elements of $V^{(1)}$ and $V^{(1)} P$ are calculated directly.

The starting point of our method is the observation, that any $3 \mathrm{~N}$ interaction and thus also its $V^{(1)}$ component in momentum space can be written as a sum of terms in the form

$$
\begin{aligned}
V^{(1)}= & f\left(\boldsymbol{q}_{1}, \boldsymbol{q}_{2}, \boldsymbol{q}_{3}\right) \hat{O}_{\text {spin }}\left(\boldsymbol{q}_{1}, \boldsymbol{q}_{2}, \boldsymbol{q}_{3}, \boldsymbol{\sigma}_{1}, \boldsymbol{\sigma}_{2}, \boldsymbol{\sigma}_{3}\right) \\
& \times \hat{O}_{\text {isospin }}\left(\boldsymbol{\tau}_{1}, \boldsymbol{\tau}_{2}, \boldsymbol{\tau}_{3}\right)
\end{aligned}
$$

where $\hat{O}_{\text {spin }}$ and $\hat{O}_{\text {isospin }}$ are the operators acting on spin and isospin degrees of freedom, respectively, which are built from the spin $\left(\boldsymbol{\sigma}_{i}\right)$ and isospin $\left(\boldsymbol{\tau}_{i}\right)$ operators of individual nucleons. Scalar factors $f\left(\boldsymbol{q}_{1}, \boldsymbol{q}_{2}, \boldsymbol{q}_{3}\right)$ and spin operators $\hat{O}_{\text {spin }}\left(\boldsymbol{q}_{1}, \boldsymbol{q}_{2}, \boldsymbol{q}_{3}, \boldsymbol{\sigma}_{1}, \boldsymbol{\sigma}_{2}, \boldsymbol{\sigma}_{3}\right)$ depend on the momentum transfers $\boldsymbol{q}_{i}$ to the nucleon $i$ which are expressed in terms of the initial and final Jacobi momenta $\boldsymbol{p}, \boldsymbol{q}$ and $\boldsymbol{p}^{\prime}$, $\boldsymbol{q}^{\prime}$, respectively, as

$$
\begin{aligned}
& \boldsymbol{q}_{1}=\boldsymbol{q}^{\prime}-\boldsymbol{q}, \quad \boldsymbol{q}_{2}=\left(\boldsymbol{p}^{\prime}-\boldsymbol{p}\right)-\frac{1}{2}\left(\boldsymbol{q}^{\prime}-\boldsymbol{q}\right) \\
& \boldsymbol{q}_{3}=-\left(\boldsymbol{p}^{\prime}-\boldsymbol{p}\right)-\frac{1}{2}\left(\boldsymbol{q}^{\prime}-\boldsymbol{q}\right)=-\left(\boldsymbol{q}_{1}+\boldsymbol{q}_{2}\right) .
\end{aligned}
$$

For example, in the $\pi-\pi$ part of the TM $3 \mathrm{~N}$ force one meets the following spin-isospin structures:

$$
\begin{aligned}
& \hat{O}_{\text {spin }}\left(\boldsymbol{q}_{1}, \boldsymbol{q}_{2}, \boldsymbol{q}_{3}, \boldsymbol{\sigma}_{1}, \boldsymbol{\sigma}_{2}, \boldsymbol{\sigma}_{3}\right)=\left(\boldsymbol{\sigma}_{2} \cdot \boldsymbol{q}_{2}\right)\left(\boldsymbol{\sigma}_{3} \cdot \boldsymbol{q}_{3}\right), \\
& \left(\boldsymbol{\sigma}_{2} \cdot \boldsymbol{q}_{2}\right)\left(\boldsymbol{\sigma}_{3} \cdot \boldsymbol{q}_{3}\right)\left(\boldsymbol{q}_{2} \cdot \boldsymbol{q}_{3}\right), \\
& \left(\boldsymbol{\sigma}_{2} \cdot \boldsymbol{q}_{2}\right)\left(\boldsymbol{\sigma}_{3} \cdot \boldsymbol{q}_{3}\right)\left(\left(\boldsymbol{q}_{2}\right)^{2}+\left(\boldsymbol{q}_{3}\right)^{2}\right), \\
& \boldsymbol{\sigma}_{1} \cdot \boldsymbol{q}_{2} \times \boldsymbol{q}_{3} \\
& \hat{O}_{i \operatorname{sospin}}\left(\boldsymbol{\tau}_{1}, \boldsymbol{\tau}_{2}, \boldsymbol{\tau}_{3}\right)=\boldsymbol{\tau}_{2} \cdot \boldsymbol{\tau}_{3}, \\
& i \boldsymbol{\tau}_{1} \cdot \boldsymbol{\tau}_{2} \times \boldsymbol{\tau}_{3} .
\end{aligned}
$$

Note, that not all combinations of $\hat{O}_{\text {spin }}$ and $\hat{O}_{\text {isospin }}$ actually appear in the above example.

In the first step of aPWD we calculate $3 \mathrm{NF}$ matrix elements using partial-wave states $|p, q, \beta\rangle[16]$ in the socalled $L S$-coupling

$|p, q, \beta\rangle_{1} \equiv\left|p q(l \lambda) L\left(s \frac{1}{2}\right) S(L S) J M_{J}\right\rangle_{1}\left|\left(t \frac{1}{2}\right) T M_{T}\right\rangle_{1}{ }^{\prime}$

where the relative orbital angular momentum $l$ (within the pair (23)) and $\lambda$ (between the pair (23) and nucleon 1) are coupled to the total orbital angular momentum $L$. In the spin space, the spin of the (23) pair is coupled with the spin $\frac{1}{2}$ of the nucleon 1 to the total spin $S$. Finally, $L$ and $S$ are coupled to the total $3 \mathrm{~N}$ angular momentum $J$ with the projection $M_{J}$. The index 1 emphasizes that the spectator particle is nucleon $1 . \beta$ describes the set of discrete quantum numbers discussed above. The isospin state is the same as in the basis state $|p, q, \alpha\rangle$.

In this basis, it is easy to decouple the isospin and spin parts from the momentum part, what leads to the following form of a $3 \mathrm{NF}$ matrix element:

$$
\begin{aligned}
& \left\langle p^{\prime} q^{\prime}\left(l^{\prime} \lambda^{\prime}\right) L^{\prime}\left(s^{\prime} \frac{1}{2}\right) S^{\prime}\left(L^{\prime} S^{\prime}\right) J M_{J}\right|\left\langle\left(t^{\prime} \frac{1}{2}\right) T^{\prime} m_{T^{\prime}}\right| \\
& \times V^{(1)}\left|p q(l \lambda) L\left(s \frac{1}{2}\right) S(L S) J M_{J}\right\rangle\left|\left(t \frac{1}{2}\right) T M_{T}\right\rangle= \\
& \int \mathrm{d} \hat{p}^{\prime} \int \mathrm{d} \hat{q}^{\prime} \int \mathrm{d} \hat{p} \int \mathrm{d} \hat{q} \\
& \sum_{m_{L^{\prime}}} C\left(L^{\prime}, S^{\prime}, J ; m_{L^{\prime}}, M_{J}-m_{L^{\prime}}, M_{J}\right) \mathcal{Y}_{l^{\prime}, \lambda^{\prime}}^{* L^{\prime}, m_{L^{\prime}}}\left(\hat{p}^{\prime}, \hat{q}^{\prime}\right) \\
& \times \sum_{m_{L}} C\left(L, S, J ; m_{L}, M_{J}-m_{L}, M_{J}\right) \mathcal{Y}_{l, \lambda}^{L, m_{L}}(\hat{p}, \hat{q}) \\
& \times\left\langle\left(s^{\prime} \frac{1}{2}\right) S^{\prime} M_{J}-m_{L^{\prime}}\right| \hat{O}_{s p i n}\left(\boldsymbol{p}^{\prime}, \boldsymbol{q}^{\prime}, \boldsymbol{p}, \boldsymbol{q}\right) \\
& \times\left|\left(s \frac{1}{2}\right) S M_{J}-m_{L}\right\rangle \\
& \times f\left(\boldsymbol{p}^{\prime}, \boldsymbol{q}^{\prime}, \boldsymbol{p}, \boldsymbol{q}\right)\left\langle\left(t^{\prime} \frac{1}{2}\right) T^{\prime} M_{T}\left|\hat{O}_{i \operatorname{sospin}}\right|\left(t \frac{1}{2}\right) T M_{T}\right\rangle,
\end{aligned}
$$

where

$$
\begin{aligned}
\mathcal{Y}_{l, \lambda}^{L, m_{L}}(\hat{p}, \hat{q}) \equiv & \sum_{\substack{m_{l}=-l\\
}}^{l} C\left(l, \lambda, L ; m_{l}, m_{L}-m_{l}, m_{L}\right) \\
& \times Y_{l, m_{l}}(\hat{p}) Y_{\lambda, m_{L}-m_{l}}(\hat{q})
\end{aligned}
$$

with the standard Clebsch-Gordan coefficients and the spherical harmonics. For abbreviation we skip in (10) and in the following the spin $\boldsymbol{\sigma}_{i}$ and the isospin $\boldsymbol{\tau}_{i}$ operators in the arguments of $\hat{O}_{\text {spin }}$ and $\hat{O}_{i \text { sospin }}$ operators.

The matrix element in the spin space appearing in $(10),\left\langle\left(s^{\prime} \frac{1}{2}\right) S^{\prime} M_{J}-m_{L^{\prime}}\left|\hat{O}_{\text {spin }}\left(\boldsymbol{p}^{\prime}, \boldsymbol{q}^{\prime}, \boldsymbol{p}, \boldsymbol{q}\right)\right|\left(s \frac{1}{2}\right) S M_{J}-\right.$ $\left.m_{L}\right\rangle$, depends on the momenta $\boldsymbol{q}_{i}$ and spin quantum numbers. Using a software for symbolic calculations (such as Mathematica $^{\circledR}[19]$ in our case) it is very easy to calculate this matrix element for all combinations of spin quantum numbers as a function of the momentum vectors $\boldsymbol{q}_{i}$. To this aim we use the Kronecker product built in Mathematica, which allows us to express the spin matrix element in terms of simple matrix operations. This is even more straightforward in the case of the isospin matrix element, which does not depend on any additional parameters. Another advantage of using a software for symbolic calculations is the possibility to generate a Fortran (or C) code in an automatized way. This eliminates possible errors which can be introduced during programming of very lengthy formulas for the spin matrix element. The calculation of the $3 \mathrm{NF}$ matrix elements requires finally an eight-dimensional integration shown in (10). In a typical case the total isospin and its projection is conserved. We also assume that the considered $3 \mathrm{~N}$ force is rotationally invariant. Then the matrix elements in (10) vanish unless $J=J^{\prime}$ and $M_{J}=M_{J^{\prime}}$, and, additionally, do not depend 
on $M_{J}$. Thus we can calculate

$$
\begin{aligned}
& G\left(l^{\prime}, \lambda^{\prime}, L^{\prime}, s^{\prime}, S^{\prime}, t^{\prime}, l, \lambda, L, s, S, J, t, T, M_{T}\right) \equiv \frac{1}{2 J+1} \\
& \times \sum_{M_{J}=-J}^{J}\left\langle\left(t^{\prime} \frac{1}{2}\right) T, M_{T}\right| \\
& \times\left\langle p^{\prime} q^{\prime}\left(l^{\prime} \lambda^{\prime}\right) L^{\prime}\left(s^{\prime} \frac{1}{2}\right) S^{\prime}\left(L^{\prime} S^{\prime}\right) J M_{J}\right| \\
& \times V^{(1)}\left|p q(l \lambda) L\left(s \frac{1}{2}\right) S(L S) J M_{J}\right\rangle\left|\left(t \frac{1}{2}\right) T, M_{T}\right\rangle,
\end{aligned}
$$

which is equal to the original matrix element of $V^{(1)}$ given in eq. (10). The integrand in $G\left(l^{\prime}, \lambda^{\prime}, L^{\prime}, s^{\prime}, S^{\prime}, t^{\prime}, l, \lambda\right.$, $\left.L, s, S, J, t, T, M_{T}\right)$, i.e.,

$$
\begin{aligned}
& \int \mathrm{d} \hat{p}^{\prime} \int \mathrm{d} \hat{q}^{\prime} \int \mathrm{d} \hat{p} \int \mathrm{d} \hat{q} \frac{1}{2 J+1} \sum_{M_{J}=-J}^{J} \sum_{m_{L^{\prime}}} \\
& C\left(L^{\prime}, S^{\prime}, J ; m_{L^{\prime}}, M_{J}-m_{L^{\prime}}, M_{J}\right) \mathcal{Y}_{l^{\prime}, \lambda^{\prime}, m_{L^{\prime}}}\left(\hat{p}^{\prime}, \hat{q}^{\prime}\right) \\
& \times \sum_{m_{L}} C\left(L, S, J ; m_{L}, M_{J}-m_{L}, M_{J}\right) \mathcal{Y}_{l, \lambda}^{L, m_{L}}(\hat{p}, \hat{q}) \\
& \times\left\langle\left(s^{\prime} \frac{1}{2}\right) S^{\prime} M_{J}-m_{L^{\prime}}\right| \hat{O}_{\text {spin }}\left(\boldsymbol{p}^{\prime}, \boldsymbol{q}^{\prime}, \boldsymbol{p}, \boldsymbol{q}\right) \\
& \times\left|\left(s \frac{1}{2}\right) S M_{J}-m_{L}\right\rangle \\
& \times f\left(\boldsymbol{p}^{\prime}, \boldsymbol{q}^{\prime}, \boldsymbol{p}, \boldsymbol{q}\right)\left\langle\left(t^{\prime} \frac{1}{2}\right) T^{\prime} M_{T}\left|\hat{O}_{i \text { sospin }}\right|\left(t \frac{1}{2}\right) T M_{T}\right\rangle,
\end{aligned}
$$

is a scalar and thus does not depend on all directions of the Jacobi momenta [20]. Therefore we are free to choose for example $\boldsymbol{p}$ along the $z$-axis $(\boldsymbol{p}=(0,0, p))$ and $\phi_{q}=0$ and thus we are left with five-fold integrations only

$$
\begin{aligned}
& G\left(l^{\prime}, \lambda^{\prime}, L^{\prime}, s^{\prime}, S^{\prime}, t^{\prime}, l, \lambda, L, s, S, J, t, T, M_{T}\right)= \\
& 8 \pi^{2} \int \mathrm{d} \hat{p}^{\prime} \int \mathrm{d} \hat{q}^{\prime} \int \mathrm{d} \cos \left(\theta_{q}\right) \frac{1}{2 J+1} \sum_{M_{J}=-J}^{J} \sum_{m_{L^{\prime}}} \\
& \quad C\left(L^{\prime}, S^{\prime}, J ; m_{L^{\prime}}, M_{J}-m_{L^{\prime}}, M_{J}\right) \mathcal{Y}_{l^{\prime}, \lambda^{\prime}}^{*}, m_{L^{\prime}}\left(\hat{p}^{\prime}, \hat{q}^{\prime}\right) \\
& \times \sum_{m_{L}} C\left(L, S, J ; m_{L}, M_{J}-m_{L}, M_{J}\right) \\
& \times \mathcal{Y}_{l, \lambda}^{L, m_{L}}\left(\hat{z}, \hat{q}=\left(\sin \left(\theta_{q}\right), 0, \cos \left(\theta_{q}\right)\right)\right) \\
& \times\left\langle\left(s^{\prime} \frac{1}{2}\right) S^{\prime} M_{J}-m_{L^{\prime}}\right| \hat{O}_{s p i n}\left(\boldsymbol{p}^{\prime}, \boldsymbol{q}^{\prime}, \boldsymbol{p}=(0,0, p),\right. \\
& \left.\boldsymbol{q}=q\left(\sin \left(\theta_{q}\right), 0, \cos \left(\theta_{q}\right)\right)\right)\left|\left(s \frac{1}{2}\right) S M_{J}-m_{L}\right\rangle \\
& \times f\left(\boldsymbol{p}^{\prime}, \boldsymbol{q}^{\prime}, \boldsymbol{p}=(0,0, p), \boldsymbol{q}=q\left(\sin \left(\theta_{q}\right), 0, \cos \left(\theta_{q}\right)\right)\right) \\
& \times\left\langle\left(t^{\prime} \frac{1}{2}\right) T M_{T}\left|\hat{O}_{i s o s p i n}\right|\left(t \frac{1}{2}\right) T M_{T}\right\rangle .
\end{aligned}
$$

The reduction of the number of integrations for a simple example of $3 \mathrm{NF}$ is numerically exemplified in ref. [11].

The remaining summations over $m_{L^{\prime}}, m_{L}$ and $M_{J}$ and five-fold integrations can be performed for a small number of $\left(p, q, p^{\prime}, q^{\prime}\right)$ combinations even on a personal computer. However, a large number of five-dimensional integrations, as they are needed to obtain all matrix elements needed for the solution of the $3 \mathrm{~N}$ Faddeev equations, has to be carried out on a powerful parallel computer. Once the matrix elements $\left\langle p^{\prime}, q^{\prime}, \beta^{\prime}\left|V^{(1)}\right| p, q, \beta\right\rangle$ are calculated, recoupling to the $j I$-representation, $\left\langle p^{\prime}, q^{\prime}, \alpha^{\prime}\left|V^{(1)}\right| p, q, \alpha\right\rangle$, can be easily performed [16]

$$
\begin{aligned}
& \left\langle p^{\prime}, q^{\prime}, \alpha^{\prime}\left|V^{(1)}\right| p, q, \alpha\right\rangle= \\
& \sum_{\beta, \beta^{\prime}} \sqrt{(2 j+1)(2 J+1)(2 L+1)(2 S+1)}\left\{\begin{array}{lll}
l & s & j \\
\lambda & \frac{1}{2} & I \\
L & S & J
\end{array}\right\} \\
& \times \sqrt{\left(2 j^{\prime}+1\right)\left(2 J^{\prime}+1\right)\left(2 L^{\prime}+1\right)\left(2 S^{\prime}+1\right)}\left\{\begin{array}{lll}
l^{\prime} & s^{\prime} & j^{\prime} \\
\lambda^{\prime} & \frac{1}{2} & I^{\prime} \\
L^{\prime} & S^{\prime} & J
\end{array}\right\} \\
& \times\left\langle p^{\prime}, q^{\prime}, \beta^{\prime}\left|V^{(1)}\right| p, q, \beta\right\rangle .
\end{aligned}
$$

Now let us turn to the $V^{(1)}(1+P)$ operator and discuss its $V^{(1)} P_{12} P_{23}$ matrix element

$$
{ }_{1}\left\langle p^{\prime}, q^{\prime}, \beta^{\prime}\left|V^{(1)} P_{12} P_{23}\right| p, q, \beta\right\rangle_{1}=\int \mathrm{d} \tilde{\boldsymbol{p}}^{\prime} \int \mathrm{d} \tilde{\boldsymbol{q}}^{\prime} \int \mathrm{d} \tilde{\boldsymbol{p}} \int \mathrm{d} \tilde{\boldsymbol{q}}
$$$$
{ }_{1}\left\langle p^{\prime}, q^{\prime}, \beta^{\prime} \mid \tilde{\boldsymbol{p}^{\prime}} \tilde{\boldsymbol{q}}^{\prime}\right\rangle\left\langle\tilde{\boldsymbol{p}^{\prime}} \tilde{\boldsymbol{q}}^{\prime}\left|V^{(1)} P_{12} P_{23}\right| \tilde{\boldsymbol{p}} \tilde{\boldsymbol{q}}\right\rangle\langle\tilde{\boldsymbol{p}} \tilde{\boldsymbol{q}} \mid p, q, \beta\rangle_{1} .
$$

Since

$$
\begin{aligned}
& P_{12} P_{23}|\tilde{\boldsymbol{p}} \tilde{\boldsymbol{q}}\rangle_{1}=\left|-\frac{1}{2} \tilde{\boldsymbol{p}}+\frac{3}{4} \tilde{\boldsymbol{q}},-\tilde{\boldsymbol{p}}-\frac{1}{2} \tilde{\boldsymbol{q}}\right\rangle_{1} \\
& \times P_{12}^{s p i n} P_{23}^{s p i n}\left|\left(s \frac{1}{2}\right) S M_{S}\right\rangle_{1} \\
& \times P_{12}^{\text {isospin }} P_{23}^{\text {isospin }}\left|\left(t \frac{1}{2}\right) T M_{T}\right\rangle_{1}, \\
& P_{12}^{s p i n} P_{23}^{s p i n}\left|\left(s \frac{1}{2}\right) S M_{S}\right\rangle_{1}=\left|\left(s \frac{1}{2}\right) S M_{S}\right\rangle_{2}= \\
& \sum_{s^{\prime \prime}}(-)^{s} \sqrt{\hat{s}^{\prime \prime} \hat{s}}\left\{\begin{array}{lll}
\frac{1}{2} & \frac{1}{2} & s^{\prime \prime} \\
\frac{1}{2} & S & s
\end{array}\right\}\left|\left(s^{\prime \prime} \frac{1}{2}\right) S M_{S}\right\rangle_{1}, \\
& P_{12}^{i s o s p i n} P_{23}^{i s o s p i n}\left|\left(t \frac{1}{2}\right) T M_{T}\right\rangle_{1}=\left|\left(t \frac{1}{2}\right) T M_{T}\right\rangle_{2}= \\
& \sum_{t^{\prime \prime}}(-)^{t} \sqrt{t^{\prime \prime} \hat{t}}\left\{\begin{array}{lll}
\frac{1}{2} & \frac{1}{2} & t^{\prime \prime} \\
\frac{1}{2} & T & t
\end{array}\right\}\left|\left(t^{\prime \prime} \frac{1}{2}\right) T M_{T}\right\rangle_{1} \text {, }
\end{aligned}
$$

where $\hat{a} \equiv 2 a+1$ and $P_{i j}^{s p i n}\left(P_{i j}^{i s o s p i n}\right)$ is the part of the 
$P_{i j}$ operator acting in the spin (isospin) space, one gets

$$
\begin{aligned}
& { }_{1}\left\langle p^{\prime}, q^{\prime}, \beta^{\prime}\left|V^{(1)} P_{12} P_{23}\right| p, q, \beta\right\rangle_{1}=\int \mathrm{d} \hat{p}^{\prime} \int \mathrm{d} \hat{q}^{\prime} \int \mathrm{d} \hat{p} \int \mathrm{d} \hat{q} \\
& \sum_{m_{L^{\prime}}} C\left(L^{\prime}, S^{\prime}, J ; m_{L^{\prime}}, M_{J}-m_{L^{\prime}}, M_{J}\right) \mathcal{Y}_{l^{\prime}, \lambda^{\prime}}^{* L^{\prime}, m_{L^{\prime}}}\left(\hat{p}^{\prime}, \hat{q}^{\prime}\right) \\
& \times \sum_{m_{L}} C\left(L, S, J ; m_{L}, M_{J}-m_{L}, M_{J}\right) \mathcal{Y}_{l, \lambda}^{L, m_{L}}(\hat{p}, \hat{q}) \\
& \times \sum_{s^{\prime \prime}}(-)^{s} \sqrt{\hat{s}^{\prime \prime} \hat{s}}\left\{\begin{array}{ccc}
\frac{1}{2} & \frac{1}{2} & s^{\prime \prime} \\
\frac{1}{2} & S & s
\end{array}\right\} \sum_{t^{\prime \prime}}(-)^{t} \sqrt{t^{\prime \prime} \hat{t}}\left\{\begin{array}{lll}
\frac{1}{2} & \frac{1}{2} & t^{\prime \prime} \\
\frac{1}{2} & T & t
\end{array}\right\} \\
& \times\left\langle\left(s^{\prime} \frac{1}{2}\right) S^{\prime} M_{J}-m_{L^{\prime}}\right. \\
& \times \hat{O}_{\text {spin }}\left(\boldsymbol{p}^{\prime}, \boldsymbol{q}^{\prime},-\frac{1}{2} \boldsymbol{p}+\frac{3}{4} \boldsymbol{q},-\boldsymbol{p}-\frac{1}{2} \boldsymbol{q}\right) \\
& \left|\left(s^{\prime \prime} \frac{1}{2}\right) S M_{J}-m_{L}\right\rangle_{1} f\left(\boldsymbol{p}^{\prime}, \boldsymbol{q}^{\prime},-\frac{1}{2} \boldsymbol{p}+\frac{3}{4} \boldsymbol{q},-\boldsymbol{p}-\frac{1}{2} \boldsymbol{q}\right) \\
& \times\left\langle\left(t^{\prime} \frac{1}{2}\right) T^{\prime} M_{T}\left|\hat{O}_{i \operatorname{sospin}}\right|\left(t^{\prime \prime} \frac{1}{2}\right) T M_{T}\right\rangle_{1} \text {. }
\end{aligned}
$$

Similarly, for $V^{(1)} P_{13} P_{23}$ one gets

$$
\begin{aligned}
& { }_{1}\left\langle p^{\prime}, q^{\prime}, \beta^{\prime}\left|V^{(1)} P_{13} P_{23}\right| p, q, \beta\right\rangle_{1}=\int \mathrm{d} \hat{p}^{\prime} \int \mathrm{d} \hat{q}^{\prime} \int \mathrm{d} \hat{p} \int \mathrm{d} \hat{q} \\
& \sum_{m_{L^{\prime}}} C\left(L^{\prime}, S^{\prime}, J ; m_{L^{\prime}}, M_{J}-m_{L^{\prime}}, M_{J}\right) \mathcal{Y}_{l^{\prime}, \lambda^{\prime}}^{*}, m_{L^{\prime}}\left(\hat{p}^{\prime}, \hat{q}^{\prime}\right) \\
& \times \sum_{m_{L}} C\left(L, S, J ; m_{L}, M_{J}-m_{L}, M_{J}\right) \mathcal{Y}_{l, \lambda}^{L, m_{L}}(\hat{p}, \hat{q}) \\
& \times \sum_{s^{\prime \prime}}(-)^{s^{\prime \prime}} \sqrt{\hat{s^{\prime \prime}} \hat{s}}\left\{\begin{array}{cc}
\frac{1}{2} & \frac{1}{2} s^{\prime \prime} \\
\frac{1}{2} & S s
\end{array}\right\} \sum_{t^{\prime \prime}}(-)^{t^{\prime \prime}} \sqrt{\hat{t}^{\prime \prime} \hat{t}}\left\{\begin{array}{cc}
\frac{1}{2} & \frac{1}{2} t^{\prime \prime} \\
\frac{1}{2} T & t
\end{array}\right\} \\
& \times\left\langle\left(s^{\prime} \frac{1}{2}\right) S^{\prime} M_{J}-m_{L^{\prime}}\right| \\
& \quad \times \hat{O}_{\text {spin }}\left(\boldsymbol{p}^{\prime}, \boldsymbol{q}^{\prime},-\frac{1}{2} \boldsymbol{p}-\frac{3}{4} \boldsymbol{q}, \boldsymbol{p}-\frac{1}{2} \boldsymbol{q}\right) \\
& \left|\left(s^{\prime \prime} \frac{1}{2}\right) S M_{J}-m_{L}\right\rangle_{1} f\left(\boldsymbol{p}^{\prime}, \boldsymbol{q}^{\prime},-\frac{1}{2} \boldsymbol{p}-\frac{3}{4} \boldsymbol{q}, \boldsymbol{p}-\frac{1}{2} \boldsymbol{q}\right) \\
& \times\left\langle\left(t^{\prime} \frac{1}{2}\right) T^{\prime} M_{T}\left|\hat{O}_{\text {isospin }}\right|\left(t^{\prime \prime} \frac{1}{2}\right) T M_{T}\right\rangle_{1} .
\end{aligned}
$$

That means that the calculation of these two contributions proceeds in the same way as calculation of the $V^{(1)}$ matrix element. Only the arguments of the term $\hat{O}_{\text {spin }}$ have to be changed and additional factors originating from the recoupling of the spin and isospin quantum numbers have to be taken into account. As for the $V^{(1)}$ operator also here the eight-fold integrations can be reduced to the five-fold ones and recalculation to $|p, q, \alpha\rangle$ states can be performed.

It is important to note that, since our basis states $|p, q, \alpha\rangle$ are antisymmetric with respect to the exchange of nucleons 2 and 3, eqs. (20) and (21) yield the same values for the matrix elements. This allows one to reduce significantly the size of the codes and the required computation time.

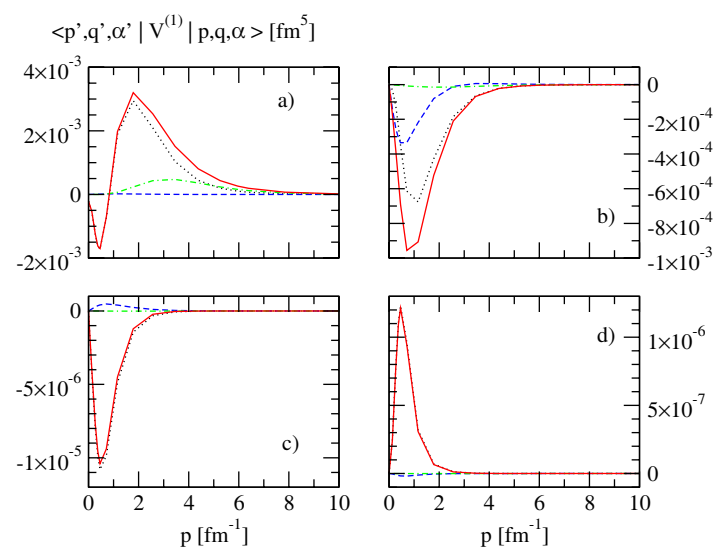

Fig. 1. (Color online) The TM $3 \mathrm{NF}$ matrix elements $\left\langle p^{\prime}=\right.$ $\left.0.132 \mathrm{fm}^{-1}, q^{\prime}=0.132 \mathrm{fm}^{-1}, \alpha^{\prime}\left|V^{(1)}\right| p, q=0.132 \mathrm{fm}^{-1}, \alpha\right\rangle$ as a function of the $p$ momentum for $\left(\alpha^{\prime}, \alpha\right)$ : a) $(1,1)$, b) $(1,4)$, c) $(6,3)$ d) $(6,8)$. The solid (red) curve represents the full TM $3 \mathrm{NF}$ and the other curves show the contributions coming from the $\pi-\pi$ (black dotted), $\pi-\rho$ (blue dashed) and $\rho-\rho$ (green dotdashed) components.

\section{Results}

\subsection{The TM 3NF and its $\pi-\pi, \pi-\rho$, and $\rho-\rho$ components}

Since the aim of this work is not to study the dependence of the matrix elements of the TM force on its parameters, in the following we use their values given in table I of ref. [4]: $a=1.03 \mu^{-1}, b=-2.62 \mu^{-3}, c=0.91 \mu^{-3}$, $d=-0.753 \mu^{-3}$ with $\mu=139.6 \mathrm{MeV}$ and $\Lambda_{N N \pi}=5.8 \mu$. In the numerical implementation of (10) we use the same number of Gaussian points for each of the five angular domains. It might be more efficient to relax this constraint in future applications and to optimize the grids further. Thus our integration method leaves room for improvement, even if we will later demonstrate in subsect. 3.7 that it leads to fully converged results.

The TM 3NF matrix elements calculated in the basis (5) are functions of four momentum magnitudes and two sets of discrete quantum numbers. In figs. 1, 2, examples of the TM force $V^{(1)}$ matrix elements are shown together with its $\pi-\pi, \pi-\rho$ and $\rho-\rho$ components in one-dimensional plots. In fig. 1, the matrix elements $\left\langle p^{\prime}, q^{\prime}, \alpha^{\prime}\left|V^{(1)}\right| p, q, \alpha\right\rangle$ for $p^{\prime}=q^{\prime}=q=0.132 \mathrm{fm}^{-1}$ and for different channel pairs $\left(\alpha^{\prime}, \alpha\right)$ (see table 1 ) are shown as a function of the momentum $p$. The same matrix elements but for the momenta $p^{\prime}=0.711 \mathrm{fm}^{-1}, q^{\prime}=0.132 \mathrm{fm}^{-1}$, and $q=2.84 \mathrm{fm}^{-1}$ are shown in fig. 2 again as a function of $p$. The $\pi-\pi$ part dominates in all cases but the $\pi-\rho$ part is also important (see figs. $1 \mathrm{~b}, 2 \mathrm{a}-\mathrm{c}$ ). The $\rho-\rho$ part is of less importance for all the considered matrix elements.

\subsection{The aPWD for $\mathrm{V}^{(1)}(1+\mathrm{P})$ operator}

As was described in sect. 2, aPWD can be applied not only to the $V^{(1)}$ alone but also to the $V^{(1)}(1+P)$ operator. Using aPWD for $V^{(1)}(1+P)$ has the same advantages 

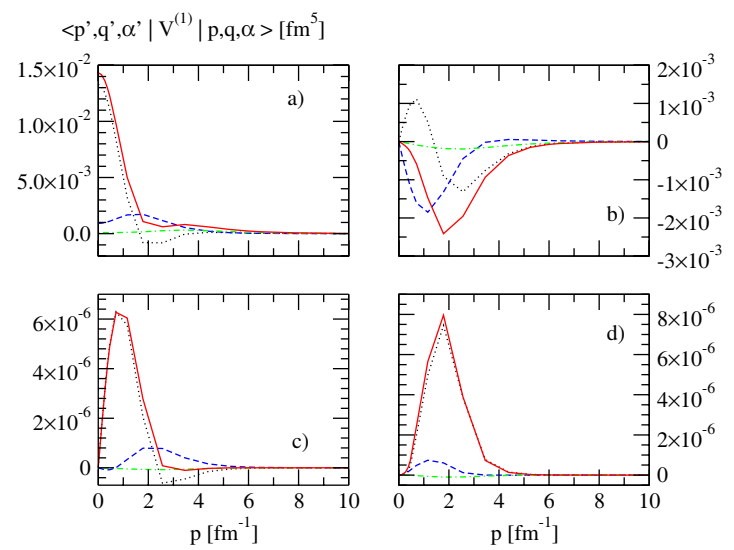

Fig. 2. (Color online) The same as in fig. 1 but for momenta values $p^{\prime}=0.711 \mathrm{fm}^{-1}, q^{\prime}=0.132 \mathrm{fm}^{-1}, q=2.842 \mathrm{fm}^{-1}$.

Table 1. The values of the discrete quantum numbers for selected $\alpha$-states (5) for the total angular momentum $J=\frac{1}{2}$ and the positive parity $\Pi=(-1)^{l+\lambda}$.

\begin{tabular}{|c|c|c|c|c|c|c|}
\hline$\alpha$ & $l$ & $s$ & $j$ & $\lambda$ & $I$ & $t$ \\
\hline 1 & 0 & 0 & 0 & 0 & $\frac{1}{2}$ & 1 \\
3 & 1 & 0 & 1 & 1 & $\frac{1}{2}$ & 0 \\
4 & 1 & 0 & 1 & 1 & $\frac{3}{2}$ & 0 \\
6 & 0 & 1 & 1 & 2 & $\frac{3}{2}$ & 0 \\
8 & 2 & 1 & 1 & 2 & $\frac{3}{2}$ & 0 \\
\hline
\end{tabular}

as for the $V^{(1)}$ operator: the automatized procedure can be easily tuned to any kind of $3 \mathrm{NF}$ and reduces the possibility of errors. In the current implementation of aPWD the calculation of $V^{(1)}(1+P)$ matrix elements needs about the one and half amount of the computing time needed for $V^{(1)}$, which is important from the practical point of view. Finally, in the standard scheme of PWD, the number of intermediate partial waves used to represent the $P$ operator is limited and might be insufficient. In the case of aPWD there is no separate decomposition of the permutation operator which corresponds to the inclusion of all three-body intermediate waves. In figs. 3 and 4 the matrix elements of $V^{(1)}(1+P)$ are shown for the same momenta and channels as in figs. 1 and 2 , respectively. For the channel combinations $(1,1)$ and $(6,3)$ in fig. 3 and $(1,1)$ and $(6,8)$ in fig. 4 , where the $\pi-\pi$ force dominates, the picture is similar to the corresponding ones in figs. 1 and 2. For the remaining channel combinations the differences are more visible, for example the inclusion of the permutation operator for the $\pi-\pi$ component for the $(6,8)$ pair in fig. 3 leads to the change of the sign and strength of this force. In that case also the $\pi-\rho$ part becomes bigger after the permutation operator is applied. Also for the $(6,3)$ case in figs. 3 and 4 the action of the permutation operator changes the strength of the matrix element and increases the momentum range, where both $\pi-\pi$ and $\pi-\rho$ components play a significant role. For the majority of the

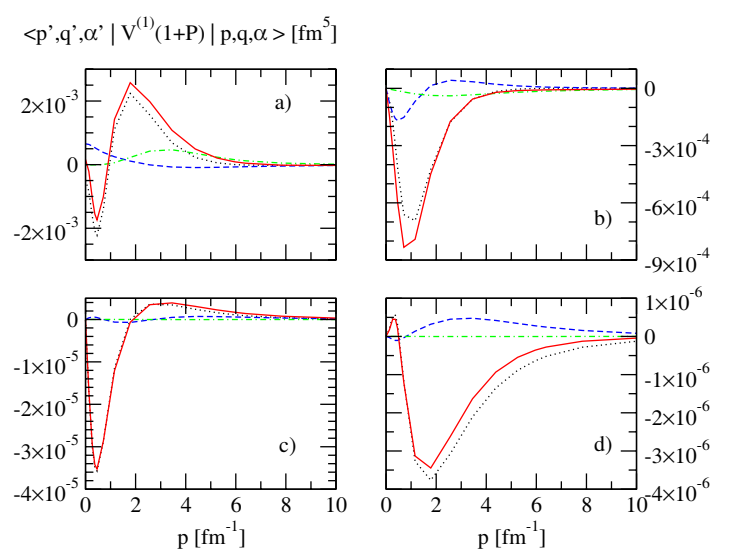

Fig. 3. (Color online) The same as in fig. 1 but for the $V^{(1)}(1+$ $P)$ operator.

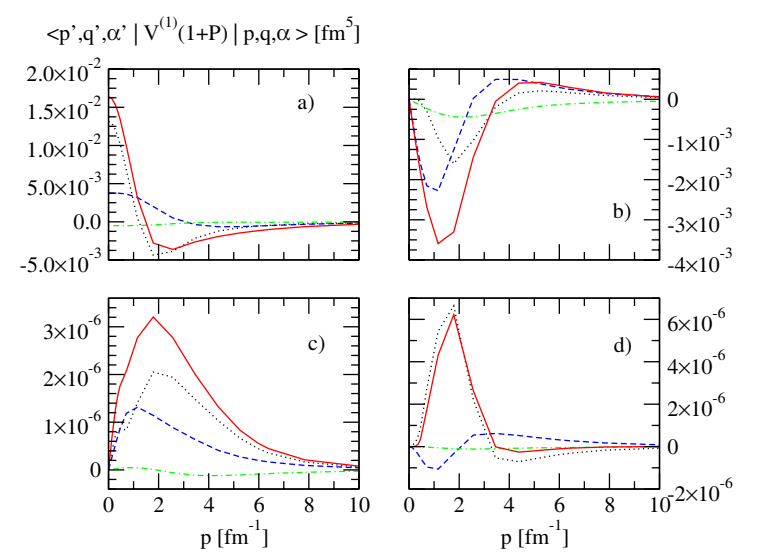

Fig. 4. (Color online) The same as in fig. 2 but for the $V^{(1)}(1+$ $P$ ) operator.

here-presented cases the $\rho$ - $\rho$ force is much smaller than the remaining interactions.

The aPWD method allows us to study the role played by different isospin structures entering the TM force. An example is given in fig. 5 where, for the $\pi$ - $\rho$ force, the contribution from the so-called "Kroll-Ruderman" and two " $\Delta$ " terms [4] (see also appendix A.1) are shown. For the presented matrix elements $\left(\left\langle p^{\prime}=0.132 \mathrm{fm}^{-1}, q^{\prime}=\right.\right.$ $\left.\left.0.132 \mathrm{fm}^{-1}, \alpha^{\prime}=1\left|V_{\pi-\rho}^{(1)}(1+P)\right| p, q=0.132 \mathrm{fm}^{-1}, \alpha=1\right\rangle\right)$ the "Kroll-Ruderman" term dominates for small momenta $p$, while the two " $\Delta$ " terms are bigger for $p>2 \mathrm{fm}^{-1}$. However, they have opposite signs, so their combined effect is weak and leads to a reduction of the strength of the dominant "Kroll-Ruderman" term.

\subsection{The comparison of the standard and automatized PWD schemes for $\pi-\pi$ and $\pi$ - $\rho$ forces}

For the $\pi-\pi$ force the partial-wave decomposition has been presented in [3] and in an alternative way in [17]. The comparison of results obtained by the aPWD and the ones obtained in ref. [17] is presented in fig. 6. Again the channel 


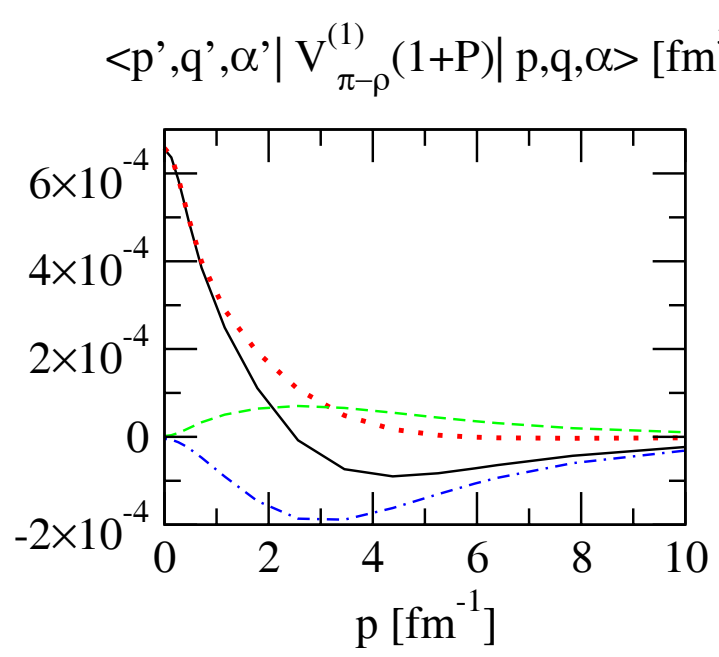

Fig. 5. (Color online) The contributions from the different parts of the $\pi-\rho$ force for matrix elements $\left\langle p^{\prime}=\right.$ $0.132 \mathrm{fm}^{-1}, \quad q^{\prime}=0.132 \mathrm{fm}^{-1}, \quad \alpha^{\prime}=1\left|V_{\pi-\rho}^{1}(1+P)\right| p, q=$ $\left.0.132 \mathrm{fm}^{-1}, \alpha=1\right\rangle$. The black solid line represents the total $\pi-\rho$ TM $3 \mathrm{NF}$ while the red dotted, green dashed and blue dot-dashed lines represent the "Kroll-Ruderman", the isospin even $\Delta$ and the isospin odd $\Delta$ terms, respectively.
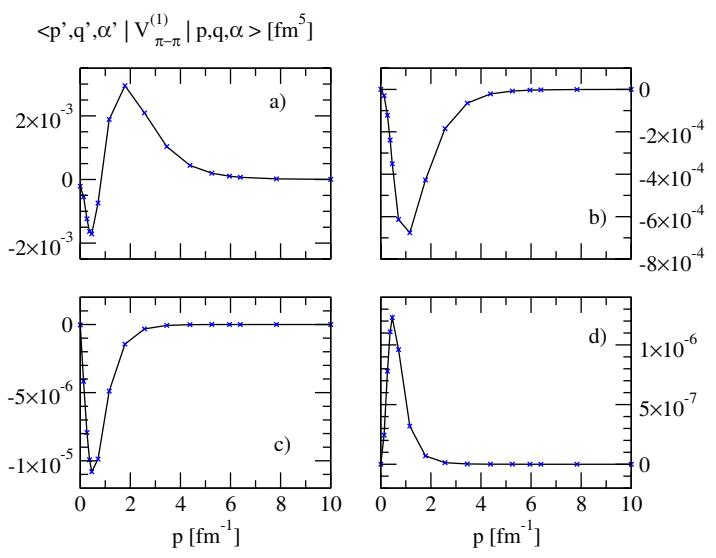

Fig. 6. (Color online) The comparison of the matrix elements of the $\pi-\pi$ force obtained in the standard (crosses) and automatized (solid line) PWD. The channel combinations and momentum values are the same as in fig. 1 .

pairs and momenta are chosen as in fig. 1. A very good agreement between both methods is clearly seen.

In appendix A we present expressions for the partialwave decomposition of the $\pi-\rho$ force. This decomposition is in the spirit of the decomposition of the $\pi-\pi$ interaction given in ref. [17]. In fig. 7 we compare the results obtained in the aPWD scheme with those based on PWD given in appendix A. Because of the internal construction of the PWD from appendix A, we compare matrix elements of $V^{(1)} P_{13} P_{23}$ instead of $V^{(1)}$. The matrix elements of the standard PWD are obtained using partial waves up to $j_{\max }=5$ in intermediate states. For this truncation, the matrix elements considered here are converged (see sect. 3.5). Again, for all given examples, the agreement between both methods is excellent.

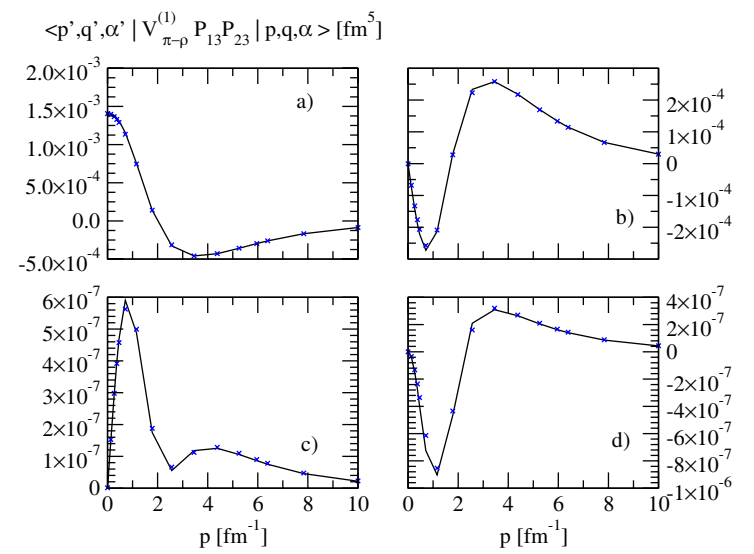

Fig. 7. (Color online) The comparison of the matrix elements of the $\pi-\rho$ force obtained in the standard PWD from appendix A (crosses) and automatized (solid line) PWD. The channels pairs and momenta are the same as in fig. 2 .

In our implementation the numerical calculation of all $18^{2}$ channels combinations of the $V^{(1)}(1+P) \pi-\rho$ $3 \mathrm{NF}$ matrix elements, on the grid of $16 p^{\prime}, q^{\prime}, p, q$ with $N=14$ Gaussian points used in each integration in 14, takes about 10 hours of CPU time when using 16000 processors of the parallel supercomputer Jugene located in Jülich, Germany. In contrast, the standard PWD requires approximately one hour on a single processor. Though the CPU time is smaller for the scheme presented in appendix A, the long time which is needed for the derivation of the partial-wave decomposition of complicated spinmomentum structures and its programming in the standard way is incomparable with the relatively short time demanded by aPWD. Another advantage of aPWD lies in its flexibility which allows one to use it easily for different operators. In the case of the standard PWD each spin-momentum structure has to be treated separately.

\subsection{The equality of $V^{(1)} P_{12} P_{23}$ and $V^{(1)} P_{13} P_{23}$}

The equality of $V^{(1)} P_{13} P_{23}$ and $V^{(1)} P_{12} P_{23}$ matrix elements between the states antisymmetrized in the (23) subsystem forms another nontrivial test of numerics. To check this, we compare some matrix elements for $V^{(1)} P_{12} P_{23}$ obtained via eq. (20) with the corresponding ones for $V^{(1)} P_{13} P_{23}$ from eq. (21). Results are displayed in fig. 8 again for four combinations of channel pairs and selected values of $p^{\prime}, q^{\prime}$ and $q$ momenta (the same as in fig. 1 ). The numerical confirmation of the equality of the $V^{(1)} P_{13} P_{23}$ and $V^{(1)} P_{12} P_{23}$ matrix elements is clear. They differ from the $V^{(1)}$ elements, as can be seen for some examples in fig. 8. All three possibilities are shown: for the channel combinations $(1,1)$ and $(1,4) V^{(1)}$ dominates, while $V^{(1)} P_{12} P_{23}$ and $V^{(1)} P_{13} P_{23}$ are much smaller. For the $(6,3)$ combination each operator gives a similar contribution to $V^{(1)}(1+P)$. For the $(6,8)$ choice and momenta around $2 \mathrm{fm}^{-1}$ the contribution from $V^{(1)}$ is much smaller than the remaining two. 

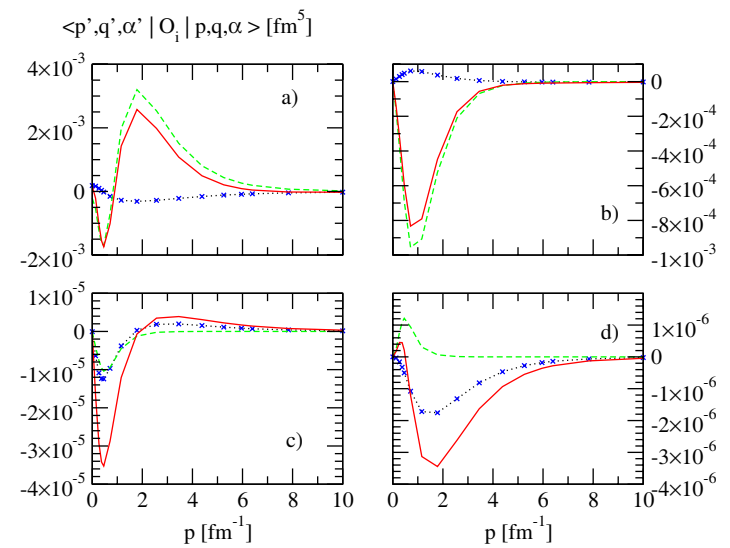

Fig. 8. (Color online) The contribution of $O_{i} \equiv V^{(1)}$, $V^{(1)} P_{13} P_{23}$ and $V^{(1)} P_{12} P_{23}$ operators to the total $V^{(1)}(1+P)$ TM 3 NF matrix elements. The channels combinations and momenta are chosen as in fig. 1 . The crosses represent $V^{(1)} P_{13} P_{23}$ matrix elements. The dashed, dotted and solid lines represent $V^{(1)}, V^{(1)} P_{12} P_{2,3}$ and $V^{(1)}(1+P)$ matrix elements, respectively.
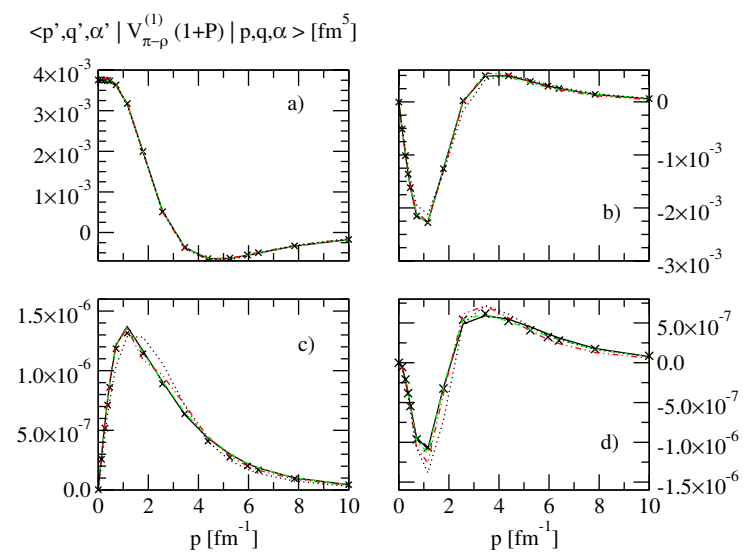

Fig. 9. (Color online) The convergence of the matrix elements of the $\pi-\rho$ part of the TM force: $V_{\pi-\rho}^{(1)}(1+P)$ with respect to the number of the intermediate partial waves used during the action of the permutation operator (see eq. (6)). The channel combinations and momenta are the same as in fig. 2 . The crosses represent predictions obtained within the aPWD approach, the dotted (black), dash-double-dotted (red), dashdotted (green) and solid (black) lines represent the results obtained with the traditional method described in appendix A with all the intermediate $3 \mathrm{~N}$ states up to $j_{\max }=2,3,4$ and 5 , respectively.

\subsection{The convergence of $V^{(1)}(1+P)$ matrix elements with respect to the number of the intermediate partial waves for the $\pi-\rho$ and the full TM forces}

The aPWD result for the $V^{(1)}(1+P)$ operator, which corresponds to the infinite number of the intermediate partial waves taken into account during the action of the permutation operator, gives the limit to which results of the traditional scheme should converge. This convergence is confirmed in figs. 9 and 10 for the $\pi-\rho$ part of the TM and the full TM $3 \mathrm{NF}$, respectively. The channels and momenta

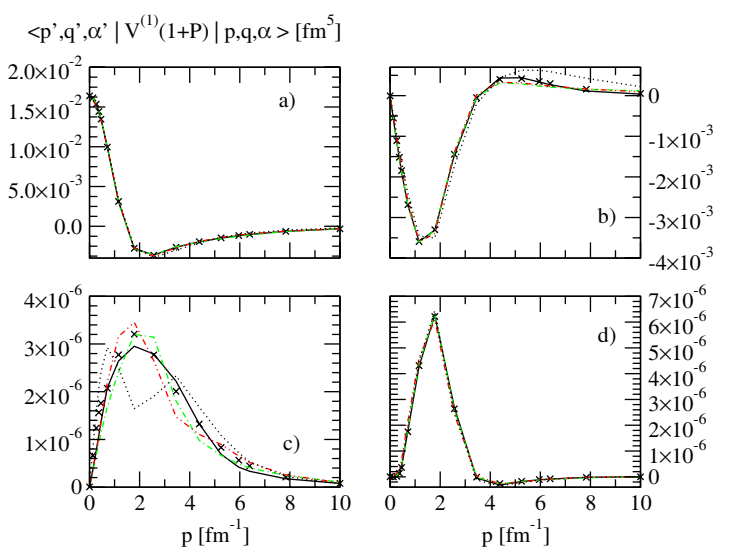

Fig. 10. (Color online) The convergence of the full TM $V^{(1)}(1+P)$ matrix elements with respect to the number of intermediate partial waves used during the action of the permutation operator. The channel combinations and momenta are as in fig. 2. The crosses represent predictions obtained within aPWD approach. The dotted (black), dash-doubledotted (red), dash-dotted (green) and solid (black) lines represent the results obtained by the action of the permutation operator on the TM $V^{(1)}$ force with all the intermediate $3 \mathrm{~N}$ states up to $j_{\max }=2,3,4$ and 5 , respectively.

are the same as in fig. 2. While for the channel combination $(1,1)$ already the smallest number of partial waves gives the aPWD limit, for the other combinations much more partial waves have to be taken into account. For one of the cases shown here (fig. 10c), taking all partial waves up to $j_{\max }=5$ is still insufficient to achieve the limit of aPWD. Note, however, that the magnitude of this matrix element is relatively small. In general, the convergence of the traditional PWD scheme is fully confirmed.

\subsection{The binding energy and correlation function for ${ }^{3} \mathrm{H}$}

As a first application, we would like to calculate in the following the binding energy of ${ }^{3} \mathrm{H}$, some energy expectation values and the correlation function. The obtained binding energies and expectation values of the kinetic energy $\left\langle H_{0}\right\rangle$, the NN potential energy $\left\langle V_{N N}\right\rangle$ and the $3 \mathrm{~N}$ potential energy $\left\langle V_{3 N}\right\rangle$ are given in table 2 for several realistic NN interactions alone and together with the TM force. The TM force was included for all states with subsystem total angular momentum $j \leq 2$. The inclusion of the TM force leads to a stronger binding of ${ }^{3} \mathrm{H}$. The binding energy changes, after the inclusion of the TM force, by approximately $-1.093 \mathrm{MeV}$ for the CDBonn potential and from -1.122 to $-1.334 \mathrm{MeV}$ for Nijmegen potentials. These results are in a reasonable agreement with the ones given in table 2 of ref. [6] for the Bonn OBEPQ $(-9.596 \mathrm{MeV})$ and the Nijmegen $(-8.689 \mathrm{MeV})$ potentials. Note, that in ref. [6] slightly different values of the $a, b$ and $c$ parameters were used. In our calculations, we include partial waves up to $j_{\max }=5$ for the two-body interaction. This is also different from ref. [6] where only partial waves up to $j_{\max }=2$ were included. Of course, for the given set of 
Table 2. The triton binding energies $E_{t}$ and the energy expectation values $\left\langle H_{0}\right\rangle,\left\langle V_{N N}\right\rangle$ and $\left\langle V_{3 N}\right\rangle$ for the different NN potentials alone and together with the TM $3 \mathrm{NF}$.

\begin{tabular}{|c|c|c|c|c|}
\hline NN potential & $E_{t}[\mathrm{MeV}]$ & $\left\langle H_{0}\right\rangle[\mathrm{MeV}]$ & $\left\langle V_{N N}\right\rangle[\mathrm{MeV}]$ & $\left\langle V_{3 N}\right\rangle[\mathrm{MeV}]$ \\
\hline CDBonn & -8.008 & 37.620 & -45.609 & - \\
Nijmegen I & -7.738 & 40.737 & -48.467 & - \\
Nijmegen II & -7.658 & 47.526 & -55.176 & - \\
Nijmegen 93 & -7.664 & 45.617 & -53.283 & - \\
\hline CDBonn + TM & -9.101 & 41.934 & -48.669 & -2.342 \\
Nijmegen I + TM & -8.860 & 45.523 & -52.277 & -2.098 \\
Nijmegen II + TM & -8.992 & 54.318 & -61.112 & -2.189 \\
Nijmegen 93 + TM & -8.841 & 51.173 & -58.092 & -1.925 \\
\hline
\end{tabular}

parameters, the binding energies do not accurately reproduce the experimental value of $-8.482 \mathrm{MeV}$. Because of the well-known scaling behavior of many N-d scattering observables with the triton binding energy (see for example $[21,22]$ ), it will be necessary to finetune the TM model such that the triton binding energy is more accurately reproduced, e.g. along the lines of ref. [13].

The inclusion of the TM $3 \mathrm{NF}$ leads for all the NN potentials to higher expectation values of the kinetic energy and lower expectation values of the NN potential energy (about $3-6 \mathrm{MeV}$ ). The expectation values of the $3 \mathrm{~N}$ potential energy amounts from $3.3 \%$ to $4.8 \%$ of the expectation values of the NN potential, depending on the particular NN potential. This observations are in line with the general expectations for the strength of $3 \mathrm{NFs}$ and the more compact state of ${ }^{3} \mathrm{H}$ when the binding energy is increased.

The correlation function is defined in the configuration space as [13]

$$
C(r) \equiv \frac{1}{3} \frac{1}{4 \pi} \int \mathrm{d} \hat{r}\left\langle\Psi\left|\sum_{i<j} \delta\left(\boldsymbol{r}-\boldsymbol{r}_{i j}\right)\right| \Psi\right\rangle,
$$

where $r_{i j}$ is the relative distance operator conjugate to the operator of the Jacobi momentum $\boldsymbol{p}$. It is shown in fig. 11 for the different NN potentials alone and combined with the TM 3NF. For the smaller distances shown in fig. 11, the probability to find two nucleons increases when the TM $3 \mathrm{NF}$ is included. At least in part, this can be understood because the correlation functions drop more quickly for larger $r$ due to the increased binding energy. Note that at short distances, the effect of the 3NFs is much smaller than the dependence on the NN interaction model. The here-presented correlation functions are in good agreement with the ones presented in [13] for the same NN potentials combined with the $\pi-\pi$ part of the TM force.

\subsection{The quality of the five-dimensional integration}

Finally, we would like to give an example of the stability of aPWD against the number of points used in the numerical integrations. In table 3 the $V^{(1)}(1+P)$ matrix elements are given for the same channels and momenta as in fig. 1 for two values of momentum $p=0.711 \mathrm{fm}^{-1}$

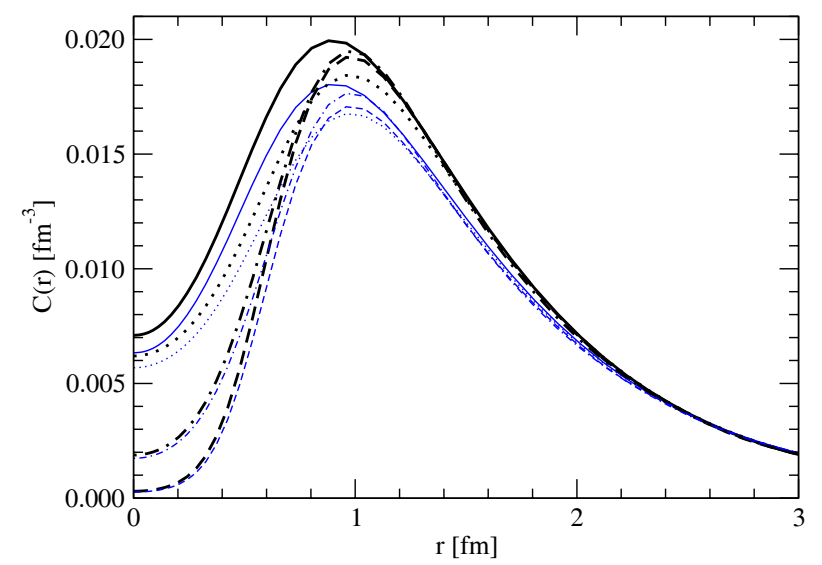

Fig. 11. (Color online) The two-body correlation function for the triton for different NN potentials alone (thin blue lines) and together with the TM 3NF (thick black lines). Results obtained with the CDBonn, Nijmegen I, Nijmegen II and Nijmegen 93 potentials are represented by solid, dotted, dashed and dashdotted curves, respectively.

and $p=5.959 \mathrm{fm}^{-1}$. Results were obtained using $N=12$ or $N=15$ Gaussian points in each of the five integrations in (14). The agreement seen in table 3 between both predictions clearly demonstrates that the numerical integration is well under control and leads to fully converged numbers.

\section{Summary}

We apply an automatized method of partial-wave decomposition to the Tucson-Melbourne three-nucleon force. The obtained results agree very well with the traditional way of a partial-wave decomposition for $\pi-\pi$ and $\pi-\rho$ contributions to the TM $3 \mathrm{NF}$. For the latter one, we also give formulas of the partial-wave decomposition in the traditional approach. Matrix elements obtained in the new way are used in the calculations of the triton wave function with different underlying nucleon-nucleon potentials. We performed also different numerical tests, which confirm the reliability of our method and computer codes. 
Table 3. The $V^{(1)}(1+P)$ matrix elements $\left(\right.$ in $\mathrm{fm}^{5}$ ) for channels combinations and momenta $p^{\prime}, q^{\prime}, q$ as in fig. 1 , depending on the number of Gaussian points $N$ used in the five-fold integration of eq. (14). The value of momentum $p$ is $0.711 \mathrm{fm}{ }^{-1}$ (left) and $5.959 \mathrm{fm}^{-1}$ (right).

\begin{tabular}{|c|c|c|c|c|}
\hline$\left(\alpha^{\prime}, \alpha\right)$ & $N=12$ & $N=15$ & $N=12$ & $N=15$ \\
& $p=0.711 \mathrm{fm}^{-1}$ & $p=0.711 \mathrm{fm}^{-1}$ & $p=5.959 \mathrm{fm}^{-1}$ & $p=5.959 \mathrm{fm}^{-1}$ \\
\hline$(1,1)$ & -0.0010139197 & -0.0010139197 & $8.9762335 \times 10^{-05}$ & $8.9762335 \times 10^{-05}$ \\
$(1,4)$ & -0.00083291615 & -0.00083291615 & $-9.0900123 \times 10^{-06}$ & $-9.0900124 \times 10^{-06}$ \\
$(6,3)$ & $-2.8739479 \times 10^{-05}$ & $-2.8739479 \times 10^{-05}$ & $1.6945111 \times 10^{-06}$ & $1.6945111 \times 10^{-06}$ \\
$(6,8)$ & $-1.212581 \times 10^{-06}$ & $-1.212580 \times 10^{-06}$ & $-3.5797036 \times 10^{-07}$ & $-3.5797045 \times 10^{-07}$ \\
\hline
\end{tabular}

Among many advantages of aPWD, we would like to emphasize its generality, efficiency, the semi-automatized process of preparing a code and the possibility of a calculation of the higher partial waves. The latter point gives hope for the future use of the full Tucson-Melbourne force in a description of $3 \mathrm{~N}$ scattering at higher energies. The expected strong effects on observables coming from a $3 \mathrm{NF}$ should be tested also for short-range parts of three-body interactions. Such parts are included in the Tucson-Melbourne force.

The automatized partial-wave decomposition is especially important in view of future applications of 3NFs arising from the $\chi \mathrm{PT}$. In this approach, consistent twoand three-body forces are derived [12]. The numerous spinmomentum and isospin structures, which occur at higher orders of the chiral expansion require an efficient and automatized method for the PWD. The here-presented results for the TM force prove that such a method already exists.

This work was supported by the Polish Ministry of Science and Higher Education under Grant No. N N202 077435. It was also partially supported by the Helmholtz Association through funds provided to the virtual institute "Spin and strong QCD" (VH-VI-231) and by the European Community-Research Infrastructure Integrating Activity "Study of Strongly Interacting Matter" (acronym HadronPhysics2, Grant Agreement n. 227431) under the Seventh Framework Programme of EU. The numerical calculations have been performed on the supercomputer cluster of the JSC, Jülich, Germany.

\section{Appendix A. Standard PWD for $\pi-\rho$ component}

The $\pi$ - $\rho$ part [6] of the Tuscon-Melbourne 3NF is given in terms of the momenta $\boldsymbol{k}_{i}$ and $\boldsymbol{k}_{i}^{\prime}$ of the individual nucleons as

$$
\begin{aligned}
& \left\langle\boldsymbol{k}_{1}^{\prime} \boldsymbol{k}_{2}^{\prime} \boldsymbol{k}_{3}^{\prime}\left|W_{1}^{\pi \rho}\right| \boldsymbol{k}_{1} \boldsymbol{k}_{2} \boldsymbol{k}_{3}\right\rangle= \\
& \frac{-1}{(2 \pi)^{6}} \frac{\delta\left(\boldsymbol{k}_{1}^{\prime}+\boldsymbol{k}_{2}^{\prime}+\boldsymbol{k}_{3}^{\prime}-\boldsymbol{k}_{1}-\boldsymbol{k}_{2}-\boldsymbol{k}_{3}\right)}{\left(q^{2}+m_{\rho}^{2}\right)\left(q^{\prime 2}+m_{\pi}^{2}\right)}\left(\sigma_{3} \cdot q^{\prime}\right) \\
& \times\left\{-\left(i \boldsymbol{\tau}_{1} \cdot \boldsymbol{\tau}_{2} \times \boldsymbol{\tau}_{3}\right) R_{K R}^{\pi \rho}\left(q^{2}, q^{2}\right)\left(i \boldsymbol{\sigma}_{1} \cdot \boldsymbol{\sigma}_{2} \times \boldsymbol{q}\right)\right. \\
& +\left(\boldsymbol{\tau}_{2} \cdot \boldsymbol{\tau}_{3}\right) R_{\Delta^{+}}^{\pi \rho}\left(q^{2}, q^{\prime 2}\right)\left(\boldsymbol{q} \times \boldsymbol{q}^{\prime}\right) \cdot\left(\boldsymbol{q} \times \boldsymbol{\sigma}_{2}\right) \\
& +\left(i \boldsymbol{\tau}_{1} \cdot \boldsymbol{\tau}_{2} \times \boldsymbol{\tau}_{3}\right) R_{\Delta^{-}}^{\pi \rho}\left(q^{2}, q^{\prime 2}\right)\left[\left(i \boldsymbol{\sigma}_{1} \cdot \boldsymbol{\sigma}_{2} \times \boldsymbol{q}^{\prime}\right) q^{2}\right. \\
& \left.\left.-\left(i \boldsymbol{\sigma}_{1} \cdot \boldsymbol{q} \times \boldsymbol{q}^{\prime}\right)\left(\boldsymbol{\sigma}_{2} \cdot \boldsymbol{q}\right)\right]\right\}+\left(2 \leftrightarrow 3, \boldsymbol{q} \leftrightarrow-\boldsymbol{q}^{\prime}\right) .
\end{aligned}
$$

Introducing isospin, $I_{+}$and $I_{-}$, and spin, $F_{K R}, F_{\Delta+}^{I}, F_{\Delta+}^{I I}$, $F_{\Delta-}^{I}$, and $F_{\Delta-}^{I I}$, operators we rewrite it as

$$
\begin{aligned}
& \left\langle\boldsymbol{k}_{1}^{\prime} \boldsymbol{k}_{2}^{\prime} \boldsymbol{k}_{3}^{\prime}\left|W_{1}^{\pi \rho}\right| \boldsymbol{k}_{1} \boldsymbol{k}_{2} \boldsymbol{k}_{3}\right\rangle= \\
& \frac{-1}{(2 \pi)^{6}} \delta\left(\boldsymbol{k}_{1}^{\prime}+\boldsymbol{k}_{2}^{\prime}+\boldsymbol{k}_{3}^{\prime}-\boldsymbol{k}_{1}-\boldsymbol{k}_{2}-\boldsymbol{k}_{3}\right) \\
& \times\left\{I_{+} R_{K R}^{\pi \rho}\left(q^{2}, q^{\prime 2}\right) F_{K R}+I_{-} R_{\Delta^{+}}^{\pi \rho}\left(q^{2}, q^{2}\right)\left(F_{\Delta^{+}}^{I}-F_{\Delta^{+}}^{I I}\right)\right. \\
& \left.+I_{+} R_{\Delta^{-}}^{\pi \rho}\left(q^{2}, q^{\prime 2}\right)\left(F_{\Delta^{-}}^{I}+F_{\Delta^{-}}^{I I}\right)\right\} \\
& +\left(2 \leftrightarrow 3, q \leftrightarrow-q^{\prime}\right),
\end{aligned}
$$

with

$$
\begin{aligned}
I_{+} & \equiv i \boldsymbol{\tau}_{1} \cdot \boldsymbol{\tau}_{2} \times \boldsymbol{\tau}_{3}, \quad I_{-} \equiv \boldsymbol{\tau}_{2} \cdot \boldsymbol{\tau}_{3} \\
F_{K R} & \equiv \frac{-\left(\boldsymbol{\sigma}_{3} \cdot \boldsymbol{q}^{\prime}\right)}{q^{\prime 2}+m_{\pi}^{2}} \frac{i \boldsymbol{\sigma}_{1} \cdot \boldsymbol{\sigma}_{2} \times \boldsymbol{q}}{q^{2}+m_{\rho}^{2}} \\
F_{\Delta^{+}} & \equiv \frac{\left(\boldsymbol{\sigma}_{3} \cdot \boldsymbol{q}^{\prime}\right)}{q^{\prime 2}+m_{\pi}^{2}} \frac{\left(\boldsymbol{q} \times \boldsymbol{q}^{\prime}\right) \cdot\left(\boldsymbol{q} \times \boldsymbol{\sigma}_{2}\right)}{q^{2}+m_{\rho}^{2}}=F_{\Delta^{+}}^{I}-F_{\Delta^{+}}^{I I} \\
F_{\Delta^{+}}^{I} & \equiv \frac{\left(\boldsymbol{\sigma}_{3} \cdot \boldsymbol{q}^{\prime}\right)\left(\boldsymbol{\sigma}_{2} \cdot \boldsymbol{q}^{\prime}\right)}{q^{\prime 2}+m_{\pi}^{2}} \frac{q^{2}}{q^{2}+m_{\rho}^{2}} \\
F_{\Delta^{+}}^{I I} & \equiv \frac{\left(\boldsymbol{\sigma}_{3} \cdot \boldsymbol{q}^{\prime}\right)}{q^{\prime 2}+m_{\pi}^{2}}\left(\boldsymbol{q}^{\prime} \cdot \boldsymbol{q}\right) \frac{\left(\boldsymbol{\sigma}_{2} \cdot \boldsymbol{q}\right)}{q^{2}+m_{\rho}^{2}} \\
F_{\Delta^{-}}^{I} & \equiv \frac{\left(\boldsymbol{\sigma}_{3} \cdot \boldsymbol{q}^{\prime}\right)\left(i \boldsymbol{\sigma}_{1} \cdot \boldsymbol{\sigma}_{2} \times \boldsymbol{q}^{\prime}\right)}{q^{\prime 2}+m_{\pi}^{2}} \frac{q^{2}}{q^{2}+m_{\rho}^{2}}
\end{aligned}
$$

and

$$
F_{\Delta^{-}}^{I I} \equiv \frac{-\left(\boldsymbol{\sigma}_{3} \cdot \boldsymbol{q}^{\prime}\right)}{q^{\prime 2}+m_{\pi}^{2}} \frac{\left(\boldsymbol{\sigma}_{2} \cdot \boldsymbol{q}\right) \cdot\left(i \boldsymbol{\sigma}_{1} \cdot \boldsymbol{q} \times \boldsymbol{q}^{\prime}\right)}{q^{2}+m_{\rho}^{2}} .
$$

The $R_{K R}^{\pi \rho}, R_{\Delta^{+}}^{\pi \rho}$, and $R_{\Delta^{-}}^{\pi \rho}$ form factors are given in terms of regularization form factors at the meson-baryon-baryon vertices $F_{i}$ as $[6]$

$$
\begin{aligned}
R_{K R}^{\pi \rho}\left(q^{2}, q^{\prime 2}\right)= & \frac{g_{\rho}^{2} g^{2}}{16 m^{3}}\left[F_{\rho N N_{D}}\left(q^{2}\right)+\kappa_{\rho} F_{\rho N N_{P}}\left(q^{2}\right)\right] \\
& \times F_{\rho N N_{D}}\left(q^{2}\right) F_{\pi N N}^{2}\left(q^{\prime 2}\right),
\end{aligned}
$$

and

$$
\begin{aligned}
& R_{\Delta^{-}}^{\pi \rho}\left(q^{2}, q^{2}\right)=\frac{1}{4} R_{\Delta^{+}}^{\pi \rho}\left(q^{2}, q^{\prime 2}\right)= \\
& \frac{1}{4} \frac{g_{\rho}}{48 m^{5}} G_{M \rho}^{*} \frac{m}{M} \frac{5 M-m}{M-m} m g^{*} g \\
& \times\left[F_{\rho N N_{D}}\left(q^{2}\right)+\kappa_{\rho} F_{\rho N N_{P}}\left(q^{2}\right)\right] \\
& \times F_{\rho N \Delta}\left(q^{2}\right) F_{\pi N \Delta}\left(q^{2}\right) F_{\pi N N}\left(q^{2}\right) .
\end{aligned}
$$


The $F_{i}$ are taken in monopole form

$$
F_{i}\left(q^{2}\right)=\frac{\Lambda_{i}^{2}-m_{b}^{2}}{\Lambda_{i}^{2}+q^{2}}
$$

with $i=\left\{\pi N N, \pi N \Delta, \rho N N_{D}, \rho N N_{P}, \rho N \Delta\right\}$. The mass of the boson at the corresponding vertex, $m_{b}$, is either $m_{\pi}$ or $m_{\rho}$ with exception of the case $i=\rho N \Delta$ when $m_{b}=0$.

We would like to have matrix elements of the threebody force in a partial-wave basis $|p q \alpha\rangle_{1}$, where $p$ and $q$ are magnitudes of Jacobi momenta ( $\boldsymbol{p}$ is the relative momentum between particles 2 and 3 and $\boldsymbol{q}$ is the momentum of the spectator particle 1 relative to the 2-3 pair) and $\alpha$ denotes discrete quantum numbers which we separate in spin, $\alpha_{J}$, and isospin, $\alpha_{T}$, parts

$$
\begin{aligned}
|p q \alpha\rangle_{1} & \equiv\left|p(l s) j q\left(\lambda \frac{1}{2}\right) I(j, I) J M ;\left(t \frac{1}{2}\right) T M_{T}\right\rangle_{1} \\
& =\left|p(l s) j q\left(\lambda \frac{1}{2}\right) I(j, I) J M\right\rangle_{1}\left|\alpha_{T}\right\rangle_{1} \\
& =\left|p q \alpha_{J}\right\rangle_{1}\left|\alpha_{T}\right\rangle_{1}
\end{aligned}
$$

The partial-wave states corresponding to different spectator nucleon $i(i=1,2,3)$ can be obtained from $|p q \alpha\rangle_{1}$ acting with proper permutation operator, for instance $P_{13} P_{23}|p q \alpha\rangle_{1}=\left|p^{\prime} q^{\prime} \alpha^{\prime}\right\rangle_{3}$.

According to the scheme presented in ref. [17] these matrix elements can be calculated as

$$
\begin{aligned}
& { }_{1}\left\langle p q \alpha\left|I_{ \pm} \mathcal{F} \mathcal{R}(1+P)\right| p^{\prime} q^{\prime} \alpha^{\prime}\right\rangle_{1}=\sum_{\alpha^{\prime \prime}} \int_{0}^{\infty} \int_{0}^{\infty} p^{\prime \prime 2} \mathrm{~d} p^{\prime \prime} q^{\prime \prime 2} \mathrm{~d} q^{\prime \prime} \\
& { }_{1}\left\langle p q \alpha\left|I_{ \pm} \mathcal{F} \mathcal{R}\right| p^{\prime \prime} q^{\prime \prime} \alpha^{\prime \prime}\right\rangle_{3}{ }_{3}\left\langle p^{\prime \prime} q^{\prime \prime} \alpha^{\prime \prime}|(1+P)| p^{\prime} q^{\prime} \alpha^{\prime}\right\rangle_{1}, \quad \text { (A.12) }
\end{aligned}
$$

with

$$
\begin{aligned}
& { }_{1}\left\langle p q \alpha\left|I_{ \pm} \mathcal{F} \mathcal{R}\right| p^{\prime \prime} q^{\prime \prime} \alpha^{\prime \prime}\right\rangle_{3}=\sum_{\alpha^{\prime \prime \prime}} \int_{0}^{\infty} \int_{0}^{\infty} p^{\prime \prime \prime 2} \mathrm{~d} p^{\prime \prime \prime} q^{\prime \prime \prime 2} \mathrm{~d} q^{\prime \prime \prime} \\
& \sum_{\dot{\alpha}} \int_{0}^{\infty} \int_{0}^{\infty} \dot{p}^{2} \mathrm{~d} \dot{p} \dot{q}^{2} \mathrm{~d} \dot{q} \\
& \times \sum_{\ddot{\alpha}} \int_{0}^{\infty} \int_{0}^{\infty} \ddot{p}^{2} \mathrm{~d} \ddot{p} \ddot{q}^{2} \mathrm{~d} \ddot{q}_{1}\left\langle p q \alpha \mid p^{\prime \prime \prime} q^{\prime \prime \prime} \alpha^{\prime \prime \prime}\right\rangle_{2} \\
& \times\left({ }_{2}\left\langle p^{\prime \prime \prime} q^{\prime \prime \prime} \alpha_{J}^{\prime \prime \prime}\left|\mathcal{F}^{(2)} \mathcal{R}^{(2)}\right| \dot{p} \dot{q} \dot{\alpha}_{J}\right\rangle_{2}{ }_{2}\left\langle\dot{p} \dot{q} \dot{\alpha}_{J} \mid \ddot{p} \ddot{q} \ddot{\alpha}_{J}\right\rangle_{3}\right. \\
& \left.\times_{3}\left\langle\ddot{p} \ddot{q} \ddot{\alpha}_{J}\left|\mathcal{F}^{(3)} \mathcal{R}^{(3)}\right| p^{\prime} q^{\prime} \alpha_{J}^{\prime}\right\rangle_{3}\right)_{2}\left\langle\alpha_{T}^{\prime \prime \prime}\left|I_{ \pm}\right| \alpha_{T}^{\prime}\right\rangle_{3},
\end{aligned}
$$

where spin operators $\mathcal{F}$ and form factors $\mathcal{R}$ are taken among $F_{K R}, F_{\Delta^{+}}^{I}, F_{\Delta^{+}}^{I I}, F_{\Delta^{-}}^{I}, F_{\Delta^{-}}^{I I}$, and $R_{K R}^{\pi \rho}, R_{\Delta^{+}}^{\pi \rho}$ and $R_{\Delta^{-}}^{\pi \rho}$, respectively, for different contributing terms.
The matrix elements of the isospin parts appearing in eq. (A.3) are given [17] by

$$
\begin{aligned}
& { }_{2}\left\langle\alpha_{T}\left|I_{-}\right| \alpha_{T}^{\prime}\right\rangle_{3}=\left\langle\left(t \frac{1}{2}\right) T M_{T}\left|\tau_{2} \cdot \tau_{3}\right|\left(t^{\prime} \frac{1}{2}\right) T^{\prime} M_{T^{\prime}}\right\rangle_{3}= \\
& \delta_{T T^{\prime}} \delta_{M_{T} M_{T^{\prime}}}(-6)(-)^{t} \sqrt{\hat{t} \hat{t}^{\prime}}\left\{\begin{array}{ccc}
\frac{1}{2} & \frac{1}{2} & t^{\prime} \\
\frac{1}{2} & 1 & \frac{1}{2} \\
t & \frac{1}{2} & T
\end{array}\right\}, \\
& { }_{2}\left\langle\alpha_{T}\left|I_{+}\right| \alpha_{T}^{\prime}\right\rangle_{3}={ }_{2}\left\langle\left(t \frac{1}{2}\right) T M_{T}\left|i \tau_{1} \cdot \tau_{2} \tau_{3}\right|\left(t^{\prime} \frac{1}{2}\right) T^{\prime} M_{T^{\prime}}\right\rangle_{3}= \\
& -\delta_{T T^{\prime}} \delta_{M_{T} M_{T^{\prime}}} 24(-)^{2 T} \sqrt{\hat{t} \hat{t}^{\prime}} \sum_{\lambda=1 / 2}^{t+1 / 2}(-)^{3 \lambda+1 / 2} \\
& \times\left\{\begin{array}{lll}
\lambda & \frac{1}{2} & 1 \\
\frac{1}{2} & \frac{1}{2} & t
\end{array}\right\}\left\{\begin{array}{ccc}
T & \frac{1}{2} & t \\
\frac{1}{2} & 1 & \lambda \\
t^{\prime} & \frac{1}{2} & \frac{1}{2}
\end{array}\right\}
\end{aligned}
$$

where we use the abbreviation $\hat{a} \equiv 2 a+1$.

In the following subsections we will present the resulting expressions for the partial-wave-decomposed matrix elements ${ }_{2}\left\langle p^{\prime \prime \prime} q^{\prime \prime \prime} \alpha_{J}^{\prime \prime \prime}\left|\mathcal{F}^{(2)} \mathcal{R}^{(2)}\right| \dot{p} \dot{q} \dot{\alpha}_{J}\right\rangle_{2}$ and ${ }_{3}\left\langle\ddot{p} \ddot{q} \ddot{\alpha}_{J}\right|$ $\mathcal{F}^{(3)} \mathcal{R}^{(3)}\left|p^{\prime} q^{\prime} \alpha_{J}^{\prime}\right\rangle_{3}$ of different contributing terms to $\pi$ - $\rho$ Tucson-Melbourne $3 \mathrm{NF}$.

\section{Appendix A.1. The Kroll-Ruderman term $\mathrm{F}_{\mathrm{KR}} \mathbf{R}_{\mathrm{KR}}^{\pi \rho}$}

The matrix elements of ${ }_{2}\left\langle p^{\prime \prime \prime} q^{\prime \prime \prime} \alpha_{J}^{\prime \prime \prime}\left|\mathcal{F}^{(2)} \mathcal{R}^{(2)}\right| \dot{p} \dot{q} \dot{\alpha}_{J}\right\rangle_{2}$ and ${ }_{3}\left\langle\ddot{p} \ddot{q} \ddot{\alpha}_{J}\left|\mathcal{F}^{(3)} \mathcal{R}^{(3)}\right| p^{\prime} q^{\prime} \alpha_{J}^{\prime}\right\rangle_{3}$ for the $F_{K R} R_{K R}^{\pi \rho}$ term are identified as

$$
\begin{aligned}
& { }_{2}\left\langle p^{\prime \prime \prime} q^{\prime \prime \prime} \alpha_{J}^{\prime \prime \prime}\left|\mathcal{F}^{(2)} \mathcal{R}^{(2)}\right| \dot{p} \dot{q} \dot{\alpha} J\right\rangle_{2} \rightarrow \\
& \frac{g_{\rho}^{2} g^{2}}{16 m^{3}}{ }^{2}\left\langle p^{\prime \prime \prime} q^{\prime \prime \prime} \alpha_{J}^{\prime \prime \prime}\left|\frac{-\left(\boldsymbol{\sigma}_{3} \cdot \boldsymbol{q}^{\prime}\right)}{q^{\prime 2}+m_{\pi}^{2}} F_{\pi N N}^{2}\left(q^{2}\right)\right| \dot{p} \dot{q} \dot{\alpha}_{J}\right\rangle_{2}, \\
& { }_{3}\left\langle\ddot{p} \ddot{q} \ddot{\alpha}_{J}\left|\mathcal{F}^{(3)} \mathcal{R}^{(3)}\right| p^{\prime} q^{\prime} \alpha_{J}^{\prime}\right\rangle_{3} \rightarrow \\
& { }_{3}\left\langle\ddot{p} \ddot{q} \ddot{\alpha}_{J}\right| \frac{i \boldsymbol{\sigma}_{1} \cdot \boldsymbol{\sigma}_{2} \times \boldsymbol{q}}{q^{2}+m_{\rho}^{2}}\left[F_{\rho N N_{D}}\left(q^{2}\right)+\kappa_{\rho} F_{\rho N N_{P}}\left(q^{2}\right)\right] \\
& \times F_{\rho N N_{D}}\left(q^{2}\right)\left|p^{\prime} q^{\prime} \alpha_{J}^{\prime}\right\rangle_{3},
\end{aligned}
$$

where $\boldsymbol{q}^{\prime}=\dot{\boldsymbol{p}}-\boldsymbol{p}^{\prime \prime \prime}$ and $\boldsymbol{q}=\boldsymbol{p}^{\prime}-\ddot{\boldsymbol{p}}$. They are given by

$$
\begin{aligned}
& \frac{g_{\rho}^{2} g^{2}}{16 m^{3}} 2\left\langle p^{\prime \prime \prime} q^{\prime \prime \prime} \alpha_{J}^{\prime \prime \prime}\left|\frac{-\left(\boldsymbol{\sigma}_{3} \cdot \boldsymbol{q}^{\prime}\right)}{q^{2}+m_{\pi}^{2}} F_{\pi N N}^{2}\left(q^{\prime 2}\right)\right| \dot{p} \dot{q} \dot{\alpha}\right\rangle_{J}= \\
& \frac{g_{\rho}^{2} g^{2}}{16 m^{3}} \frac{\delta\left(q^{\prime \prime \prime}-\dot{q}\right)}{q^{\prime \prime \prime 2}} \delta_{j^{\prime \prime \prime} j} \delta_{\lambda^{\prime \prime \prime} \dot{\lambda}} \delta_{I^{\prime \prime \prime} \dot{I}} \delta_{J^{\prime \prime \prime} \dot{j}} \delta_{M^{\prime \prime \prime} \dot{M}} \delta_{\left|l^{\prime \prime \prime}-i\right|, 1}
\end{aligned}
$$

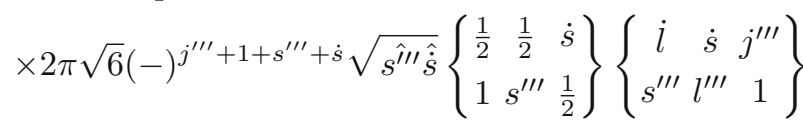

$$
\begin{aligned}
& \times \sqrt{\max \left(l^{\prime \prime \prime}, i\right)} \\
& \times\left(p^{\prime \prime \prime} H_{i}^{\pi N N}\left(p^{\prime \prime \prime}, \dot{p}\right)-\dot{p} H_{l}^{\pi N N}\left(p^{\prime \prime \prime}, \dot{p}\right)\right)(-)^{\max \left(l^{\prime \prime \prime}, i\right)},
\end{aligned}
$$


and

$$
\begin{aligned}
& { }_{3}\left\langle\ddot{p} \ddot{q} \ddot{\alpha}_{J}\right| \frac{i \boldsymbol{\sigma}_{1} \cdot \boldsymbol{\sigma}_{2} \times \boldsymbol{q}}{q^{2}+m_{\rho}^{2}}\left[F_{\rho N N_{D}}\left(q^{2}\right)+\kappa_{\rho} F_{\rho N N_{P}}\left(q^{2}\right)\right] \\
& \times F_{\rho N N_{D}}\left(q^{2}\right)\left|p^{\prime} q^{\prime} \alpha_{J}^{\prime}\right\rangle_{3}= \\
& \frac{\delta\left(\ddot{q}-q^{\prime}\right)}{q^{2}} \delta_{\ddot{I} I^{\prime}} \delta_{\ddot{\lambda} \lambda^{\prime}} \delta_{\ddot{j} j^{\prime}} \\
& \times(-)^{1+\ddot{j}+s^{\prime}} 12 \sqrt{6} \pi \sqrt{\hat{l} \hat{l}} \hat{i}\left\{\begin{array}{ccc}
l^{\prime} & s^{\prime} & \ddot{j} \\
\ddot{s} & \ddot{l} & 1
\end{array}\right\} \\
& \times\left(\begin{array}{lll}
l^{\prime} & 1 & \ddot{l} \\
0 & 0 & 0
\end{array}\right) \sqrt{\hat{s}^{\prime} \hat{\ddot{s}}}\left\{\begin{array}{ccc}
1 & 1 & 1 \\
\frac{1}{2} & \frac{1}{2} & s^{\prime} \\
\frac{1}{2} & \frac{1}{2} & \ddot{s}
\end{array}\right\} \\
& \times\left(\ddot{p} H_{l^{\prime}}^{K R, \rho N N}\left(p^{\prime}, \ddot{p}\right)-p^{\prime} H_{\ddot{l}}^{K R, \rho N N}\left(p^{\prime}, \ddot{p}\right)\right),
\end{aligned}
$$

with

$$
\begin{aligned}
& H_{l}^{\pi N N}\left(p^{\prime \prime \prime}, \dot{p}\right)=\frac{1}{p^{\prime \prime \prime} \dot{p}}\left(Q_{l}\left(B_{m_{\pi}}\right)-Q_{l}\left(B_{\Lambda_{\pi N N}}\right)\right) \\
& +\frac{\Lambda_{\pi N N}^{2}-m_{\pi}^{2}}{2\left(p^{\prime \prime \prime} \dot{p}\right)^{2}} Q_{l}^{\prime}\left(B_{\Lambda_{\pi N N}}\right), \\
& H_{l}^{K R, \rho N N}\left(p^{\prime}, \ddot{p}\right)=\frac{1}{p^{\prime} \ddot{p}}\left(Q_{l}\left(B_{m_{\rho}}\right)-Q_{\bar{l}}\left(B_{\Lambda_{\rho N N_{D}}}\right)\right) \\
& +\frac{\Lambda_{\rho N N_{D}}^{2}-m_{\rho}^{2}}{2\left(p^{\prime} \ddot{p}\right)^{2}} Q_{l}^{\prime}\left(B_{\Lambda_{\rho N N_{D}}}\right) \\
& +\frac{\kappa_{\rho}\left(Q_{l}\left(B_{m_{\rho}}\right)-\frac{\Lambda_{\rho N N_{P}}^{2}-m_{\rho}^{2}}{p^{\prime} \ddot{p}} Q_{\rho N N_{P}}^{2}-\Lambda_{\rho N N_{D}}^{2}\left(B_{\Lambda_{\rho N N_{D}}}\right)\right.}{\left.+\frac{\Lambda_{\rho N N_{D}}^{2}-m_{\rho}^{2}}{\Lambda_{\rho N N_{P}}^{2}-\Lambda_{\rho N N_{D}}^{2}} Q_{l}\left(B_{\Lambda_{\rho N N_{P}}}\right)\right),}
\end{aligned}
$$

and

$$
\begin{aligned}
& B_{m_{\pi}}=\frac{p^{\prime \prime \prime 2}+\dot{p}^{2}+m_{\pi}^{2}}{2 p^{\prime \prime \prime} \dot{p}}, \quad B_{\Lambda_{\pi N N}}=\frac{p^{\prime \prime \prime 2}+\dot{p}^{2}+\Lambda_{\pi N N}^{2}}{2 p^{\prime \prime \prime} \dot{p}}, \\
& B_{m_{\rho}}=\frac{p^{\prime 2}+\ddot{p}^{2}+m_{\rho}^{2}}{2 p^{\prime} \ddot{p}}, \quad B_{\Lambda_{\rho N N_{D}}}=\frac{p^{\prime 2}+\ddot{p}^{2}+\Lambda_{\rho N N_{D}}^{2}}{2 p^{\prime} \ddot{p}}, \\
& B_{\Lambda_{\rho N N_{P}}}=\frac{p^{\prime 2}+\ddot{p}^{2}+\Lambda_{\rho N N_{P}}^{2}}{2 p^{\prime} \ddot{p}} .
\end{aligned}
$$

The $Q_{l}(x)$ are Legendre functions of the second kind.

\section{Appendix A.2. The term $\mathrm{F}_{\Delta+}^{\prime} \mathbf{R}_{\Delta+}^{\pi \rho}$}

The term $F_{\Delta+}^{I}$ is written as

$$
\begin{aligned}
F_{\Delta+}^{I}= & \frac{\left(\boldsymbol{\sigma}_{3} \cdot \boldsymbol{q}^{\prime}\right)\left(\boldsymbol{\sigma}_{2} \cdot \boldsymbol{q}^{\prime}\right)}{q^{\prime 2}+m_{\pi}^{2}} \frac{q^{2}}{q^{2}+m_{\rho}^{2}}=\sum_{\mu}(-)^{\mu} \\
& \times\left\{\sqrt{\frac{4 \pi}{3}} \frac{q^{\prime} Y_{1}^{-\mu}\left(\hat{q}^{\prime}\right)\left(\sigma_{3} \cdot q^{\prime}\right)}{q^{\prime 2}+m_{\pi}^{2}}\right\}\left\{\frac{\sigma_{2}^{\mu} q^{2}}{q^{2}+m_{\rho}^{2}}\right\},
\end{aligned}
$$

and the matrix elements of the $-\mu$ component of ${ }_{2}\left\langle p^{\prime \prime \prime} q^{\prime \prime \prime} \alpha_{J}^{\prime \prime \prime}\left|\mathcal{F}^{(2)} \mathcal{R}^{(2)}\right| \dot{p} \dot{q} \dot{\alpha}_{J}\right\rangle_{2}$ and of the $\mu$ component of ${ }_{3}\left\langle\ddot{p} \ddot{q} \ddot{\alpha}_{J}\left|\mathcal{F}^{(3)} \mathcal{R}^{(3)}\right| p^{\prime} q^{\prime} \alpha_{J}^{\prime}\right\rangle_{3}$ for $F_{\Delta+}^{I} R_{\Delta+}^{\pi \rho}$ are identified as

$\left\{{ }_{2}\left\langle p^{\prime \prime \prime} q^{\prime \prime \prime} \alpha_{J}^{\prime \prime \prime}\left|\mathcal{F}^{(2)} \mathcal{R}^{(2)}\right| \dot{p} \dot{q} \dot{\alpha}_{J}\right\rangle_{2}\right\}^{-\mu} \rightarrow$

$\frac{g_{\rho} g}{48 m^{5}} G_{M \rho}^{*} \frac{m}{M} \frac{5 M-m}{M-m} m g_{2}^{*}\left\langle p^{\prime \prime \prime} q^{\prime \prime \prime} \alpha_{J}^{\prime \prime \prime}\right|$

$\times \sqrt{\frac{4 \pi}{3}} \frac{q^{\prime} Y_{1}^{-\mu}\left(\hat{q}^{\prime}\right)\left(\sigma_{3} \cdot q^{\prime}\right)}{q^{\prime 2}+m_{\pi}^{2}} F_{\pi N N}\left(q^{\prime 2}\right) F_{\pi N \Delta}\left(q^{\prime 2}\right)|\dot{p} \dot{q} \dot{\alpha} J\rangle_{2}$,

$\left\{{ }_{3}\left\langle\ddot{p} \ddot{q} \ddot{\alpha}_{J}\left|\mathcal{F}^{(3)} \mathcal{R}^{(3)}\right| p^{\prime} q^{\prime} \alpha_{J}^{\prime}\right\rangle_{3}\right\}^{\mu} \rightarrow$

${ }_{3}\left\langle\dddot{p} \ddot{q} \ddot{\alpha}_{J}\right| \frac{\sigma_{2}^{\mu} q^{2}}{q^{2}+m_{\rho}^{2}}\left[F_{\rho N N_{D}}\left(q^{2}\right)+\kappa_{\rho} F_{\rho N N_{P}}\left(q^{2}\right)\right]$

$\times F_{\rho N \Delta}\left(q^{2}\right)\left|p^{\prime} q^{\prime} \alpha_{J}^{\prime}\right\rangle_{3}$,

where $\boldsymbol{q}^{\prime}=\dot{\boldsymbol{p}}-\boldsymbol{p}^{\prime \prime \prime}$ and $\boldsymbol{q}=\boldsymbol{p}^{\prime}-\ddot{\boldsymbol{p}}$. They are given by

$$
\begin{aligned}
& { }_{2}\left\langle p^{\prime \prime \prime} q^{\prime \prime \prime} \alpha_{J}^{\prime \prime \prime}\right| \sqrt{\frac{4 \pi}{3}} \frac{q^{\prime} Y_{1}^{-\mu}\left(\hat{q}^{\prime}\right)\left(\sigma_{3} \cdot \boldsymbol{q}^{\prime}\right)}{q^{\prime 2}+m_{\pi}^{2}} F_{\pi N N}\left(q^{\prime 2}\right) \\
& \times F_{\pi N \Delta}\left(q^{\prime 2}\right)|\dot{p} \dot{q} \dot{\alpha}\rangle_{2}= \\
& \frac{\delta\left(q^{\prime \prime \prime}-\dot{q}\right)}{q^{\prime \prime \prime 2}} \delta_{\lambda^{\prime \prime \prime} \dot{\lambda}} \delta_{I^{\prime \prime \prime} \dot{I}} C\left(1-\mu \dot{J} \dot{M}, J^{\prime \prime \prime} M^{\prime \prime \prime}\right) \\
& \times(-)^{I^{\prime \prime \prime}+J^{\prime \prime \prime}} \sqrt{\hat{j^{\prime \prime \prime}} \hat{\dot{j}} \hat{s}^{\prime \prime \prime} \hat{\dot{s}} \hat{\dot{J}}}\left\{\begin{array}{ccc}
\frac{1}{2} & \frac{1}{2} & \dot{s} \\
1 & s^{\prime \prime \prime} & \frac{1}{2}
\end{array}\right\}\left\{\begin{array}{ccc}
1 & \dot{j} & j^{\prime \prime \prime} \\
I^{\prime \prime \prime} & J^{\prime \prime \prime} & \dot{J}
\end{array}\right\} \\
& \times\left[\delta_{l^{\prime \prime \prime}} i \frac{2 \pi}{3} \sqrt{6}(-)^{l^{\prime \prime \prime}+1} \tilde{H}_{l^{\prime \prime \prime}}^{\pi N N-\pi N \Delta}\left(p^{\prime \prime \prime}, \dot{p}\right)\left\{\begin{array}{ccc}
j^{\prime \prime \prime} & \dot{j} & 1 \\
\dot{s} & s^{\prime \prime \prime} & l^{\prime \prime \prime}
\end{array}\right\}\right. \\
& -40 \pi \sqrt{6}(-)^{s^{\prime \prime \prime}+\dot{j}}\left\{\begin{array}{ccc}
2 & 1 & 1 \\
i & \dot{s} & j \\
l^{\prime \prime \prime} & s^{\prime \prime \prime} & j^{\prime \prime \prime}
\end{array}\right\} \\
& \times \sum_{\bar{l}} \hat{\bar{l}} H_{\bar{l}}^{\pi N N-\pi N \Delta}\left(p^{\prime \prime \prime}, \dot{p}\right) \sum_{a+b=2} \frac{p^{\prime \prime \prime} \dot{p}^{b}}{\sqrt{(2 a) !(2 b) !}} \\
& \left.\times\left\{\begin{array}{ccc}
b & a & 2 \\
l^{\prime \prime \prime} & i & \bar{l}
\end{array}\right\} C\left(a 0 \bar{l} 0, l^{\prime \prime \prime} 0\right) C(b 0 \bar{l} 0, i j 0)\right],
\end{aligned}
$$

and

$$
\begin{aligned}
& { }_{3}\left\langle\ddot{p} \ddot{q} \ddot{\alpha}_{J}\right| \sigma_{2}^{\mu} \frac{q^{2}}{q^{2}+m_{\rho}^{2}}\left[F_{\rho N N_{D}}\left(q^{2}\right)+\kappa_{\rho} F_{\rho N N_{P}}\left(q^{2}\right)\right] \\
& \times F_{\rho N \Delta}\left(q^{2}\right)\left|p^{\prime} q^{\prime} \alpha^{\prime}\right\rangle_{3}= \\
& \frac{\delta\left(\ddot{q}-q^{\prime}\right)}{q^{\prime 2}} \delta_{\ddot{l} l^{\prime}} \delta_{\ddot{\lambda} \lambda^{\prime}} \delta_{\ddot{I} I^{\prime}} \sqrt{6} 2 \pi \tilde{H}_{l^{\prime}}^{\rho N}\left(\ddot{p}, p^{\prime}\right)
\end{aligned}
$$

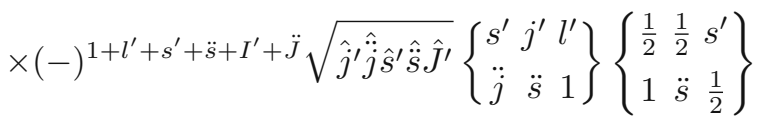

$$
\begin{aligned}
& \times\left\{\begin{array}{ccc}
1 & j^{\prime} & \ddot{j} \\
I^{\prime} & \ddot{J} & J^{\prime}
\end{array}\right\} C\left(1 \mu J^{\prime} M^{\prime}, \ddot{J} \ddot{M}\right)
\end{aligned}
$$


with

$$
\begin{aligned}
& \tilde{H}_{l}^{\pi N N-\pi N \Delta}\left(p^{\prime \prime \prime}, \dot{p}\right)=\frac{\left(\Lambda_{\pi N N}^{2}-m_{\pi}^{2}\right)\left(\Lambda_{\pi N \Delta}^{2}-m_{\pi}^{2}\right)}{p^{\prime \prime \prime} \dot{p}} \\
& \times\left(\frac{m_{\pi}^{2}}{\left(\Lambda_{\pi N \Delta}^{2}-m_{\pi}^{2}\right)\left(m_{\pi}^{2}-\Lambda_{\pi N N}^{2}\right)} Q_{l}\left(B_{m_{\pi}}\right)\right. \\
& +\frac{\Lambda_{\pi N N}^{2}}{\left(m_{\pi}^{2}-\Lambda_{\pi N N}^{2}\right)\left(\Lambda_{\pi N N}^{2}-\Lambda_{\pi N \Delta}^{2}\right)} Q_{l}\left(B_{\Lambda_{\pi N N}}\right) \\
& \left.+\frac{\Lambda_{\pi N \Delta}^{2}}{\left(\Lambda_{\pi N N}^{2}-\Lambda_{\pi N \Delta}^{2}\right)\left(\Lambda_{\pi N \Delta}^{2}-m_{\pi}^{2}\right)} Q_{l}\left(B_{\Lambda_{\pi N \Delta}}\right)\right), \\
& H_{\bar{l}}^{\pi N N-\pi N \Delta}\left(p^{\prime \prime \prime}, \dot{p}\right)= \\
& \frac{1}{p^{\prime \prime \prime} \dot{p}}\left(Q_{\bar{l}}\left(B_{m_{\pi}}\right)+\frac{\Lambda_{\pi N \Delta}^{2}-m_{\pi}^{2}}{\Lambda_{\pi N N}^{2}-\Lambda_{\pi N \Delta}^{2}} Q_{\bar{l}}\left(B_{\Lambda_{\pi N N}}\right)\right. \\
& \left.+\frac{\Lambda_{\pi N N}^{2}-m_{\pi}^{2}}{\Lambda_{\pi N \Delta}^{2}-\Lambda_{\pi N N}^{2}} Q_{\bar{l}}\left(B_{\Lambda_{\pi N \Delta}}\right)\right),
\end{aligned}
$$

and

$$
\begin{aligned}
& \tilde{H}_{\bar{l}}^{\rho N N}\left(p^{\prime}, \ddot{p}\right)=\frac{\left(\Lambda_{\rho N N_{D}}^{2}-m_{\rho}^{2}\right) \Lambda_{\rho N \Delta}^{2}}{p^{\prime} \ddot{p}} \\
& +\left(\frac{m_{\rho}^{2}}{\left(\Lambda_{\rho N \Delta}^{2}-m_{\rho}^{2}\right)\left(m_{\rho}^{2}-\Lambda_{\rho N N_{D}}^{2}\right)} Q_{\bar{l}}\left(B_{m_{\rho}}\right)\right. \\
& +\frac{\Lambda_{\rho N N_{D}}^{2}}{\left(m_{\rho}^{2}-\Lambda_{\rho N N_{D}}^{2}\right)\left(\Lambda_{\rho N N_{D}}^{2}-\Lambda_{\rho N \Delta}^{2}\right)} Q_{\bar{l}}\left(B_{\Lambda_{\rho N N_{D}}}\right) \\
& \left.+\frac{\Lambda_{\rho N \Delta}^{2}}{\left(\Lambda_{\rho N N_{D}}^{2}-\Lambda_{\rho N \Delta}^{2}\right)\left(\Lambda_{\rho N \Delta}^{2}-m_{\rho}^{2}\right)} Q_{\bar{l}}\left(B_{\Lambda_{\rho N \Delta}}\right)\right) \\
& +\frac{\left(\kappa_{\rho}\right)\left(\Lambda_{\rho N N_{P}}^{2}-m_{\rho}^{2}\right) \Lambda_{\rho N \Delta}^{2}}{p^{\prime} \ddot{p}} \\
& \times\left(\frac{m_{\rho}^{2}}{\left(\Lambda_{\rho N \Delta}^{2}-m_{\rho}^{2}\right)\left(m_{\rho}^{2}-\Lambda_{\rho N N_{P}}^{2}\right)} Q_{\bar{l}}\left(B_{m_{\rho}}\right)\right. \\
& +\frac{\Lambda_{\rho N N_{P}}^{2}}{\left(m_{\rho}^{2}-\Lambda_{\rho N N_{P}}^{2}\right)\left(\Lambda_{\rho N N_{P}}^{2}-\Lambda_{\rho N \Delta}^{2}\right)} Q_{\bar{l}}\left(B_{\Lambda_{\rho N N_{P}}}\right) \\
& \left.+\frac{\Lambda_{\rho N \Delta}^{2}}{\left(\Lambda_{\rho N N_{P}}^{2}-\Lambda_{\rho N \Delta}^{2}\right)\left(\Lambda_{\rho N \Delta}^{2}-m_{\rho}^{2}\right)} Q_{\bar{l}}\left(B_{\Lambda_{\rho N \Delta}}\right)\right) .
\end{aligned}
$$

The $B_{\Lambda_{\pi N \Delta}}$ and $B_{\Lambda_{\rho} N \Delta}$ are given by

$$
\begin{aligned}
B_{\Lambda_{\pi N \Delta}} & =\frac{p^{\prime \prime \prime 2}+\dot{p}^{2}+\Lambda_{\pi N \Delta}^{2}}{2 p^{\prime \prime \prime} \dot{p}}, \\
B_{\Lambda_{\rho N \Delta}} & =\frac{p^{\prime \prime \prime 2}+\dot{p}^{2}+\Lambda_{\rho N \Delta}^{2}}{2 p^{\prime \prime \prime} \dot{p}} .
\end{aligned}
$$

The summation over $\mu$ in eq. (A.21) can be carried through resulting in

$$
\begin{gathered}
\sum_{\mu}(-)^{\mu} C(1-\mu \dot{J} \dot{M}, J M) C\left(1 \mu J^{\prime} M^{\prime}, \dot{J} \dot{M}\right)= \\
\delta_{J J^{\prime}} \delta_{M M^{\prime}}(-)^{\dot{J}-J} \sqrt{\frac{\hat{\dot{J}}}{\hat{J}}} \cdot
\end{gathered}
$$

\section{Appendix A.3. The term $\mathrm{F}_{\Delta+}^{\prime \prime} \mathbf{R}_{\Delta+}^{\pi \rho}$}

The term $F_{\Delta+}^{I I}$ is written as

$$
\begin{aligned}
& F_{\Delta+}^{I I}=\frac{\left(\boldsymbol{\sigma}_{3} \cdot \boldsymbol{q}^{\prime}\right)}{q^{\prime 2}+m_{\pi}^{2}}\left(\boldsymbol{q} \cdot \boldsymbol{q}^{\prime}\right) \frac{\left(\boldsymbol{\sigma}_{2} \cdot \boldsymbol{q}\right)}{q^{2}+m_{\rho}^{2}}=\sum_{\mu}(-)^{\mu} \\
& \times\left\{\sqrt{\frac{4 \pi}{3}} \frac{q^{\prime} Y_{1}^{-\mu}\left(\hat{q}^{\prime}\right)\left(\boldsymbol{\sigma}_{3} \cdot \boldsymbol{q}^{\prime}\right)}{q^{\prime 2}+m_{\pi}^{2}}\right\} \\
& \times\left\{\sqrt{\frac{4 \pi}{3}} \frac{q Y_{1}^{\mu}(\hat{q})\left(\boldsymbol{\sigma}_{2} \cdot \boldsymbol{q}\right)}{q^{2}+m_{\rho}^{2}}\right\} .
\end{aligned}
$$

The matrix elements of the $-\mu$ component of ${ }_{2}\left\langle p^{\prime \prime \prime} q^{\prime \prime \prime}\right.$ $\left.\alpha_{J}^{\prime \prime \prime}\left|\mathcal{F}^{(2)} \mathcal{R}^{(2)}\right| \dot{p} \dot{q} \dot{\alpha}_{J}\right\rangle_{2}$ and of the $\mu$ component of ${ }_{3}\left\langle\ddot{p} \ddot{q} \ddot{\alpha}_{J}\right|$ $\mathcal{F}^{(3)} \mathcal{R}^{(3)}\left|p^{\prime} q^{\prime} \alpha_{J}^{\prime}\right\rangle_{3}$ for $F_{\Delta+}^{I I} R_{\Delta+}^{\pi \rho}$ are identified as

$\left\{{ }_{2}\left\langle p^{\prime \prime \prime} q^{\prime \prime \prime} \alpha_{J}^{\prime \prime \prime}\left|\mathcal{F}^{(2)} \mathcal{R}^{(2)}\right| \dot{p} \dot{q} \dot{\alpha}_{J}\right\rangle_{2}\right\}^{-\mu} \rightarrow$

$\frac{g_{\rho} g}{48 m^{5}} G_{M \rho}^{*} \frac{m}{M} \frac{5 M-m}{M-m} m g_{2}^{*}\left\langle p^{\prime \prime \prime} q^{\prime \prime \prime} \alpha_{J}^{\prime \prime \prime}\right|$

$\sqrt{\frac{4 \pi}{3}} \frac{q^{\prime} Y_{1}^{-\mu}\left(\hat{q}^{\prime}\right)\left(\boldsymbol{\sigma}_{3} \cdot \boldsymbol{q}^{\prime}\right)}{q^{\prime 2}+m_{\pi}^{2}} F_{\pi N N}\left(q^{\prime 2}\right) F_{\pi N \Delta}\left(q^{\prime 2}\right)\left|\dot{p} \dot{q} \dot{\alpha}_{J}\right\rangle_{2}$,

$\left\{{ }_{3}\left\langle\ddot{p} \ddot{q} \ddot{\alpha}_{J}\left|\mathcal{F}^{(3)} \mathcal{R}^{(3)}\right| p^{\prime} q^{\prime} \alpha_{J}^{\prime}\right\rangle_{3}\right\}^{\mu} \rightarrow$

${ }_{3}\left\langle\ddot{p} \ddot{q} \ddot{\alpha}_{J}\right| \sqrt{\frac{4 \pi}{3}} \frac{q Y_{1}^{\mu}(\hat{q})\left(\boldsymbol{\sigma}_{3} \cdot \boldsymbol{q}\right)}{q^{2}+m_{\rho}^{2}}\left[F_{\rho N N_{D}}\left(q^{2}\right)+\kappa_{\rho} F_{\rho N N_{P}}\left(q^{2}\right)\right]$

$\times F_{\rho N \Delta}\left(q^{2}\right)\left|p^{\prime} q^{\prime} \alpha_{J}^{\prime}\right\rangle_{3}$,

where $\boldsymbol{q}^{\prime}=\dot{\boldsymbol{p}}-\boldsymbol{p}^{\prime \prime \prime}$ and $\boldsymbol{q}=\boldsymbol{p}^{\prime}-\ddot{\boldsymbol{p}}$. The first term in eq. (A.31) is equal to eq. (A.23) and the second is given by

$$
\begin{aligned}
& { }_{3}\left\langle\ddot{p} \ddot{q} \ddot{\alpha}_{J}\right| \sqrt{\frac{4 \pi}{3}} \frac{q Y_{1}^{\mu}(\hat{q})\left(\boldsymbol{\sigma}_{3} \cdot \boldsymbol{q}\right)}{q^{2}+m_{\rho}^{2}}\left[F_{\rho N N_{D}}\left(q^{2}\right)+\kappa_{\rho} F_{\rho N N_{P}}\left(q^{2}\right)\right] \\
& \times F_{\rho N \Delta}\left(q^{2}\right)\left|p^{\prime} q^{\prime} \alpha_{J}^{\prime}\right\rangle_{3}= \\
& \frac{\delta\left(q^{\prime}-\ddot{q}\right)}{q^{\prime 2}} \delta_{\lambda^{\prime} \ddot{\lambda}} \delta_{I^{\prime} \ddot{I}} C\left(1 \mu J^{\prime} M^{\prime}, \ddot{J} \ddot{M}\right)
\end{aligned}
$$

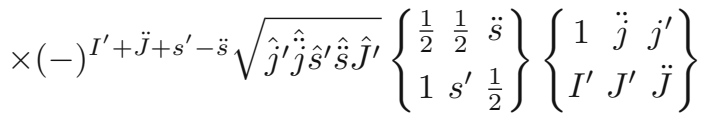

$$
\begin{aligned}
& \times\left[\delta_{l^{\prime}} \frac{2 \pi}{3} \sqrt{6}(-)^{l^{\prime}+1} \tilde{H}_{l^{\prime}}^{\rho N N}\left(p^{\prime}, \ddot{p}\right)\left\{\begin{array}{lll}
j^{\prime} & \ddot{j} & 1 \\
\ddot{s} & s^{\prime} & l^{\prime}
\end{array}\right\}\right. \\
& -40 \pi \sqrt{6}(-)^{s^{\prime}+\ddot{j}}\left\{\begin{array}{ccc}
2 & 1 & 1 \\
\ddot{l} & \ddot{s} & \ddot{j} \\
l^{\prime} & s^{\prime} & j^{\prime}
\end{array}\right\}
\end{aligned}
$$$$
\times \sum_{\bar{l}} \hat{\bar{l}} H_{\bar{l}}^{\rho N}\left(p^{\prime}, \ddot{p}\right) \sum_{a+b=2} \frac{p^{\prime a} \ddot{p}^{b}}{\sqrt{(2 a) !(2 b) !}}\left\{\begin{array}{lll}
b & a & 2 \\
l^{\prime} & \ddot{l} & \bar{l}
\end{array}\right\}
$$

$\left.\times C\left(a 0 \bar{l} 0, l^{\prime} 0\right) C(b 0 \bar{l} 0, \ddot{l} 0)\right]$, 
with

$$
\begin{aligned}
& H_{\bar{l}}^{\rho N N}\left(p^{\prime}, \ddot{p}\right)= \\
& \quad \frac{1}{p^{\prime} \ddot{p}}\left(\frac{\Lambda_{\rho N \Delta}^{2}}{\Lambda_{\rho N \Delta}^{2}-m_{\rho}^{2}} Q_{\bar{l}}\left(B_{m_{\rho}}\right)\right. \\
& +\frac{\Lambda_{\rho N \Delta}^{2}}{\Lambda_{\rho N N_{D}}^{2}-\Lambda_{\rho N \Delta}^{2}} Q_{\bar{l}}\left(B_{\Lambda_{\rho N N_{D}}}\right) \\
& \left.+\frac{\Lambda_{\rho N N_{D}}^{2}-m_{\rho}^{2}}{\Lambda_{\rho N \Delta}^{2}-m_{\rho}^{2}} \frac{\Lambda_{\rho N \Delta}^{2}}{\Lambda_{\rho N \Delta}^{2}-\Lambda_{\rho N N_{D}}^{2}} Q_{\bar{l}}\left(B_{\Lambda_{\rho N \Delta}}\right)\right) \\
& +\frac{\kappa_{\rho}}{p^{\prime} \ddot{p}}\left(\frac{\Lambda_{\rho N \Delta}^{2}}{\Lambda_{\rho N \Delta}^{2}-m_{\rho}^{2}} Q_{\bar{l}}\left(B_{m_{\rho}}\right)\right. \\
& +\frac{\Lambda_{\rho N \Delta}^{2}}{\Lambda_{\rho N N_{P}}^{2}-\Lambda_{\rho N \Delta}^{2}} Q_{\bar{l}}\left(B_{\Lambda_{\rho N N_{P}}}\right) \\
& \left.+\frac{\Lambda_{\rho N N_{P}}^{2}-m_{\rho}^{2}}{\Lambda_{\rho N \Delta}^{2}-m_{\rho}^{2}} \frac{\Lambda_{\rho N \Delta}^{2}}{\Lambda_{\rho N \Delta}^{2}-\Lambda_{\rho N N_{P}}^{2}} Q_{\bar{l}}\left(B_{\Lambda_{\rho N \Delta}}\right)\right) .
\end{aligned}
$$

\section{Appendix A.4. The term $\mathrm{F}_{\Delta-}^{\prime} \mathbf{R}_{\Delta-}^{\pi \rho}$}

Using the identity

$$
\boldsymbol{\sigma}_{1} \times \boldsymbol{\sigma}_{2} \cdot \boldsymbol{q}^{\prime}=i \sqrt{2} \sqrt{\frac{4 \pi}{3}} q^{\prime} \sum_{\mu}(-)^{\mu} \sigma_{2}^{\mu}\left\{\boldsymbol{\sigma}_{1}, Y_{1}\left(\hat{q}^{\prime}\right)\right\}^{1,-\mu},
$$

the term $F_{\Delta-}^{I}$ is written as

$$
\begin{aligned}
& F_{\Delta^{-}}^{I} \equiv \frac{\left(\boldsymbol{\sigma}_{3} \cdot \boldsymbol{q}^{\prime}\right)\left(i \boldsymbol{\sigma}_{1} \cdot \boldsymbol{\sigma}_{2} \times \boldsymbol{q}^{\prime}\right)}{q^{2}+m_{\pi}^{2}} \frac{q^{2}}{q^{2}+m_{\rho}^{2}}=\sqrt{2} \sqrt{\frac{4 \pi}{3}} \\
& \quad \times \sum_{\mu}(-)^{\mu}\left\{\frac{\left(\boldsymbol{\sigma}_{3} \cdot q^{\prime}\right) q^{\prime}\left\{\boldsymbol{\sigma}_{1}, Y_{1}\left(\hat{q}^{\prime}\right)\right\}^{1,-\mu}}{q^{\prime 2}+m_{\pi}^{2}}\right\}\left\{\frac{\sigma_{2}^{\mu} q^{2}}{q^{2}+m_{\rho}^{2}}\right\} .
\end{aligned}
$$

The matrix elements of the $-\mu$ component of ${ }_{2}\left\langle p^{\prime \prime \prime} q^{\prime \prime \prime}\right.$ $\left.\alpha_{J}^{\prime \prime \prime}\left|\mathcal{F}^{(2)} \mathcal{R}^{(2)}\right| \dot{p} \dot{q} \dot{\alpha}_{J}\right\rangle_{2}$ and of the $\mu$ component of ${ }_{3}\left\langle\ddot{p} \ddot{q} \ddot{\alpha}_{J}\right|$ $\mathcal{F}^{(3)} \mathcal{R}^{(3)}\left|p^{\prime} q^{\prime} \alpha_{J}^{\prime}\right\rangle_{3}$ for $F_{\Delta-}^{I} R_{\Delta-}^{\pi \rho}$ are identified as

$$
\begin{aligned}
& \left\{{ }_{2}\left\langle p^{\prime \prime \prime} q^{\prime \prime \prime} \alpha_{J}^{\prime \prime \prime}\left|\mathcal{F}^{(2)} \mathcal{R}^{(2)}\right| \dot{p} \dot{q} \dot{\alpha} J\right\rangle_{2}\right\}^{-\mu} \rightarrow \\
& \frac{1}{4} \frac{g_{\rho} g}{48 m^{5}} G_{M \rho}^{*} \frac{m}{M} \frac{5 M-m}{M-m} m g^{*} \\
& \times_{2}\left\langle p^{\prime \prime \prime} q^{\prime \prime \prime} \alpha_{J}^{\prime \prime \prime}\right| \sqrt{2} \sqrt{\frac{4 \pi}{3}} \frac{q^{\prime}\left\{\boldsymbol{\sigma}_{1}, Y_{1}\left(\hat{q}^{\prime}\right)\right\}^{1,-\mu}\left(\boldsymbol{\sigma}_{3} \cdot \boldsymbol{q}^{\prime}\right)}{q^{\prime 2}+m_{\pi}^{2}} \\
& \times F_{\pi N N}\left(q^{\prime 2}\right) F_{\pi N \Delta}\left(q^{\prime 2}\right)\left|\dot{p} \dot{q} \dot{\alpha}_{J}\right\rangle_{2}, \\
& \left\{{ }_{3}\left\langle\ddot{p} \ddot{q} \ddot{\alpha}_{J}\left|\mathcal{F}^{(3)} \mathcal{R}^{(3)}\right| p^{\prime} q^{\prime} \alpha_{J}^{\prime}\right\rangle_{3}\right\}^{\mu} \rightarrow \\
& { }_{3}\left\langle\ddot{p} \ddot{q} \ddot{\alpha}{ }_{J}\right| \frac{\sigma_{2}^{\mu} q^{2}}{q^{2}+m_{\rho}^{2}}\left[F_{\rho N N_{D}}\left(q^{2}\right)+\kappa_{\rho} F_{\rho N N_{P}}\left(q^{2}\right)\right] \\
& \times F_{\rho N \Delta}\left(q^{2}\right)\left|p^{\prime} q^{\prime} \alpha_{J}^{\prime}\right\rangle_{3},
\end{aligned}
$$

where $\boldsymbol{q}^{\prime}=\dot{\boldsymbol{p}}-\boldsymbol{p}^{\prime \prime \prime}$ and $\boldsymbol{q}=\boldsymbol{p}^{\prime}-\ddot{\boldsymbol{p}}$. The last term is identical to the last term in eq. (A.22) for $F_{\Delta+}^{I}$. The matrix element in the first term of eq. (A.36) is given by

$$
\begin{aligned}
& { }_{2}\left\langle p^{\prime \prime \prime} q^{\prime \prime \prime} \alpha_{J}^{\prime \prime \prime}\right| \sqrt{2} \sqrt{\frac{4 \pi}{3}} \frac{q^{\prime}\left\{\boldsymbol{\sigma}_{1}, Y_{1}\left(\hat{q}^{\prime}\right)\right\}^{1,-\mu}\left(\boldsymbol{\sigma}_{3} \cdot \boldsymbol{q}^{\prime}\right)}{q^{2}+m_{\pi}^{2}} \\
& \times F_{\pi N N}\left(q^{\prime 2}\right) F_{\pi N \Delta}\left(q^{\prime 2}\right)|\dot{p} \dot{q} \dot{\alpha} J\rangle_{2}= \\
& (-) \frac{\delta\left(q^{\prime \prime \prime}-\dot{q}\right)}{q^{\prime \prime \prime}} \delta_{\lambda^{\prime \prime \prime} \dot{\lambda}} \delta_{I^{\prime \prime \prime} \dot{I}}(-)^{I^{\prime \prime \prime}+J^{\prime \prime \prime}+1} \sqrt{\hat{j} \dot{j}^{\prime \prime \prime} \hat{j} \hat{s}^{\prime \prime \prime} \hat{\dot{s}} \hat{\dot{J}}} \\
& \times C(1-\mu \dot{J} \dot{M}, J M)\left\{\begin{array}{ccc}
1 & \dot{j} & j^{\prime \prime \prime} \\
I^{\prime \prime \prime} & J^{\prime \prime \prime} & \dot{J}
\end{array}\right\} \\
& \times\left[\delta_{l^{\prime \prime \prime} i} 4 \pi \sqrt{6}(-)^{l^{\prime \prime \prime}+s^{\prime \prime \prime}+1} \tilde{H}_{l^{\prime \prime \prime}}^{\pi N N-\pi N \Delta}\left(p^{\prime \prime \prime}, \dot{p}\right)\right. \\
& \times\left\{\begin{array}{ccc}
l^{\prime \prime \prime} & s^{\prime \prime \prime} & j^{\prime \prime \prime} \\
1 & \dot{j} & \dot{s}
\end{array}\right\}\left\{\begin{array}{ccc}
1 & 1 & 1 \\
\frac{1}{2} & \frac{1}{2} & \dot{s} \\
\frac{1}{2} & \frac{1}{2} & s^{\prime \prime \prime}
\end{array}\right\} \\
& +240 \pi \sqrt{6}(-)^{j} \sum_{\bar{l}} \hat{\bar{l}} H_{\bar{l}}^{\pi N N-\pi N \Delta}\left(p^{\prime \prime \prime}, \dot{p}\right) \sum_{a+b=2} \frac{p^{\prime \prime \prime a} \dot{p}^{b}}{\sqrt{(2 a) !(2 b) !}} \\
& \times\left\{\begin{array}{lll}
a & b & 2 \\
i & l^{\prime \prime \prime} & \bar{l}
\end{array}\right\} C\left(a 0 \bar{l} 0, l^{\prime \prime \prime} 0\right) C(b 0 \bar{l} 0, i j 0)
\end{aligned}
$$$$
\left.\times \sum_{k} \hat{k}\left\{\begin{array}{lll}
2 & k & 1 \\
1 & 1 & 1
\end{array}\right\}\left\{\begin{array}{ccc}
2 & k & 1 \\
i & \dot{s} & \dot{j} \\
l^{\prime \prime \prime} & s^{\prime \prime \prime} & j^{\prime \prime \prime}
\end{array}\right\}\left\{\begin{array}{ccc}
1 & 1 & k \\
\frac{1}{2} & \frac{1}{2} & \dot{s} \\
\frac{1}{2} & \frac{1}{2} & s^{\prime \prime \prime}
\end{array}\right\}\right] .
$$

\section{Appendix A.5. The term $\mathrm{F}_{\Delta-}^{\prime \prime} \mathrm{R}_{\Delta-}^{\pi \rho}$}

Using the identity

$$
\boldsymbol{\sigma}_{1} \cdot \boldsymbol{q} \times \boldsymbol{q}^{\prime}=-i \sqrt{2} \frac{4 \pi}{3} q q^{\prime} \sum_{\mu}(-)^{\mu}\left\{\sigma_{1}, Y_{1}(\hat{q})\right\}^{1,-\mu} Y_{1}^{\mu}\left(\hat{q}^{\prime}\right)
$$

the term $F_{\Delta-}^{I I}$ is written as

$$
\begin{aligned}
F_{\Delta^{-}}^{I I} \equiv & \frac{-\left(\boldsymbol{\sigma}_{3} \cdot \boldsymbol{q}^{\prime}\right)}{q^{\prime 2}+m_{\pi}^{2}} \frac{\left(\boldsymbol{\sigma}_{2} \cdot \boldsymbol{q}\right)\left(i \boldsymbol{\sigma}_{1} \cdot \boldsymbol{q} \times \boldsymbol{q}^{\prime}\right)}{q^{2}+m_{\rho}^{2}}= \\
& -\sqrt{2} \frac{4 \pi}{3} \sum_{\mu}(-)^{\mu}\left\{\frac{\left(\boldsymbol{\sigma}_{3} \cdot \boldsymbol{q}^{\prime}\right) q^{\prime} Y_{1}^{\mu}\left(\hat{q}^{\prime}\right)}{q^{\prime 2}+m_{\pi}^{2}}\right\} \\
& \times\left\{\frac{\left(\boldsymbol{\sigma}_{2} \cdot \boldsymbol{q}\right) q\left\{\boldsymbol{\sigma}_{1}, Y_{1}^{\mu}(\hat{q})\right\}^{1,-\mu}}{q^{2}+m_{\rho}^{2}}\right\} .
\end{aligned}
$$

The matrix elements of the $-\mu$ component of ${ }_{2}\left\langle p^{\prime \prime \prime} q^{\prime \prime \prime}\right.$ $\left.\alpha_{J}^{\prime \prime \prime}\left|\mathcal{F}^{(2)} \mathcal{R}^{(2)}\right| \dot{p} \dot{q} \dot{\alpha}_{J}\right\rangle_{2}$ and of the $\mu$ component of ${ }_{3}\left\langle\ddot{p} \ddot{q} \ddot{\alpha}_{J}\right|$ 
$\mathcal{F}^{(3)} \mathcal{R}^{(3)}\left|p^{\prime} q^{\prime} \alpha_{J}^{\prime}\right\rangle_{3}$ for $F_{\Delta-}^{I I} R_{\Delta-}^{\pi \rho}$ are identified as

$\left\{{ }_{2}\left\langle p^{\prime \prime \prime} q^{\prime \prime \prime} \alpha_{J}^{\prime \prime \prime}\left|\mathcal{F}^{(2)} \mathcal{R}^{(2)}\right| \dot{p} \dot{q} \dot{\alpha}_{J}\right\rangle_{2}\right\}^{-\mu} \rightarrow$

$-\frac{1}{4} \frac{g_{\rho} g}{48 m^{5}} G_{M \rho}^{*} \frac{m}{M} \frac{5 M-m}{M-m} m g_{2}^{*}\left\langle p^{\prime \prime \prime} q^{\prime \prime \prime} \alpha_{J}^{\prime \prime \prime}\right|$

$\sqrt{\frac{4 \pi}{3}} \frac{\left(\boldsymbol{\sigma}_{3} \cdot \boldsymbol{q}^{\prime}\right) q^{\prime} Y_{1}^{-\mu}\left(\hat{q}^{\prime}\right)}{q^{\prime 2}+m_{\pi}^{2}} F_{\pi N N}\left(q^{\prime 2}\right) F_{\pi N \Delta}\left(q^{\prime 2}\right)\left|\dot{p} \dot{q} \dot{\alpha}_{J}\right\rangle_{2}$,

$\left\{{ }_{3}\left\langle\ddot{p} \ddot{q} \ddot{\alpha}_{J}\left|\mathcal{F}^{(3)} \mathcal{R}^{(3)}\right| p^{\prime} q^{\prime} \alpha_{J}^{\prime}\right\rangle_{3}\right\}^{\mu} \rightarrow$

$\sqrt{2} \sqrt{\frac{4 \pi}{3}} 3\left\langle\ddot{p} \ddot{q} \ddot{\alpha}_{J}\right| \frac{\left(\boldsymbol{\sigma}_{2} \cdot \boldsymbol{q}\right) q\left\{\boldsymbol{\sigma}_{1}, Y_{1}(\hat{q})\right\}^{1, \mu}}{q^{2}+m_{\rho}^{2}}$

$\times\left[F_{\rho N N_{D}}\left(q^{2}\right)+\kappa_{\rho} F_{\rho N N_{P}}\left(q^{2}\right)\right] F_{\rho N \Delta}\left(q^{2}\right)\left|p^{\prime} q^{\prime} \alpha_{J}^{\prime}\right\rangle_{3}$,

where $\boldsymbol{q}^{\prime}=\dot{\boldsymbol{p}}-\boldsymbol{p}^{\prime \prime \prime}$ and $\boldsymbol{q}=\boldsymbol{p}^{\prime}-\ddot{\boldsymbol{p}}$. They are given by

$$
\begin{aligned}
& { }_{2}\left\langle p^{\prime \prime \prime} q^{\prime \prime \prime} \alpha_{J}^{\prime \prime \prime}\right| \sqrt{\frac{4 \pi}{3}} \frac{\left(\boldsymbol{\sigma}_{3} \cdot \boldsymbol{q}^{\prime}\right) q^{\prime} Y_{1}^{-\mu}\left(\hat{q}^{\prime}\right)}{q^{\prime 2}+m_{\pi}^{2}} F_{\pi N N}\left(q^{2}\right) \\
& \times F_{\pi N \Delta}\left(q^{\prime 2}\right)\left|\dot{p} \dot{q} \dot{\alpha}_{J}\right\rangle_{2}= \\
& \frac{\delta\left(q^{\prime \prime \prime}-\dot{q}\right)}{q^{\prime \prime \prime 2}} \delta_{\lambda^{\prime \prime \prime} \dot{\lambda}} \delta_{I^{\prime \prime \prime} \dot{I}} C\left(1-\mu \dot{J} \dot{M}, J^{\prime \prime \prime} M^{\prime \prime \prime}\right) \\
& \times(-)^{I^{\prime \prime \prime}+J^{\prime \prime \prime}} \sqrt{\hat{j}^{\prime \prime \prime} \hat{\dot{j}} \hat{s}^{\prime \prime \prime} \hat{\dot{s}} \hat{\dot{J}}}\left\{\begin{array}{ccc}
\frac{1}{2} & \frac{1}{2} & \dot{s} \\
1 & s^{\prime \prime \prime} & \frac{1}{2}
\end{array}\right\}\left\{\begin{array}{ccc}
1 & \dot{j} & j^{\prime \prime \prime} \\
I^{\prime \prime \prime} & J^{\prime \prime \prime} & \dot{J}
\end{array}\right\} \\
& \times\left[\delta_{l^{\prime \prime \prime}} i \frac{2 \pi}{3} \sqrt{6}(-)^{l^{\prime \prime \prime}+1} \tilde{H}_{l}^{\pi N N-\pi N \Delta}\left(p^{\prime \prime \prime}, \dot{p}\right)\left\{\begin{array}{ccc}
j^{\prime \prime \prime} & \dot{j} & 1 \\
\dot{s} & s^{\prime \prime \prime} & l^{\prime \prime \prime}
\end{array}\right\}\right. \\
& -40 \pi \sqrt{6}(-)^{s^{\prime \prime \prime}+\dot{j}}\left\{\begin{array}{ccc}
2 & 1 & 1 \\
i & \dot{s} & j \\
l^{\prime \prime \prime} & s^{\prime \prime \prime} & j^{\prime \prime \prime}
\end{array}\right\} \\
& \times \sum_{\bar{l}} \hat{\bar{l}} H_{\bar{l}}^{\pi N N-\pi N \Delta}\left(p^{\prime \prime \prime}, \dot{p}\right) \sum_{a+b=2} \frac{p^{\prime \prime \prime a} \dot{p}^{b}}{\sqrt{(2 a) !(2 b) !}} \\
& \left.\times\left\{\begin{array}{ccc}
b & a & 2 \\
l^{\prime \prime \prime} & i & \bar{l}
\end{array}\right\} C\left(a 0 \bar{l} 0, l^{\prime \prime \prime} 0\right) C(b 0 \bar{l} 0, i j 0)\right],
\end{aligned}
$$

and

$$
\begin{aligned}
& \sqrt{2} \sqrt{\frac{4 \pi}{3}}{ }_{3}\left\langle\ddot{p} \ddot{q} \ddot{\alpha}_{J}\right| \frac{\left(\boldsymbol{\sigma}_{2} \cdot \boldsymbol{q}\right) q\left\{\boldsymbol{\sigma}_{1}, Y_{1}(\hat{q})\right\}^{1, \mu}}{q^{2}+m_{\rho}^{2}} \\
& \times\left[F_{\rho N N_{D}}\left(q^{2}\right)+\kappa_{\rho} F_{\rho N N_{P}}\left(q^{2}\right)\right] F_{\rho N \Delta}\left(q^{2}\right)\left|p^{\prime} q^{\prime} \alpha_{J}^{\prime}\right\rangle_{3}= \\
& \frac{\delta\left(q^{\prime}-\ddot{q}\right)}{q^{\prime 2}} \delta_{\lambda^{\prime} \ddot{\lambda}} \delta_{I^{\prime}} \ddot{I}
\end{aligned}
$$

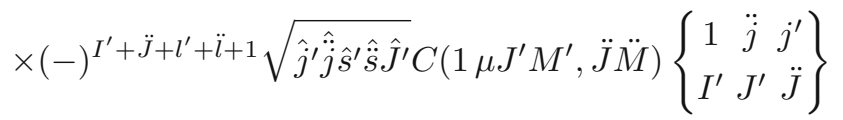

$$
\begin{aligned}
& \times\left[\delta_{l^{\prime}} 4 \pi \sqrt{6}(-)^{l^{\prime}+s^{\prime}}\left\{\begin{array}{ccc}
l^{\prime} & s^{\prime} & j^{\prime} \\
1 & \ddot{j} & \ddot{s}
\end{array}\right\}\left\{\begin{array}{ccc}
1 & 1 & 1 \\
\frac{1}{2} & \frac{1}{2} & \ddot{s} \\
\frac{1}{2} & \frac{1}{2} & s^{\prime}
\end{array}\right\} \tilde{H}_{l^{\prime}}^{\rho N N}\left(p^{\prime}, \ddot{p}\right)\right.
\end{aligned}
$$

$$
\begin{aligned}
& +240 \pi \sqrt{6}(-)^{\ddot{j}} \sum_{k}(-)^{k} \hat{k}\left\{\begin{array}{lll}
2 & k & 1 \\
1 & 1 & 1
\end{array}\right\}\left\{\begin{array}{lll}
2 & k & 1 \\
\ddot{l} & \ddot{s} & \ddot{j} \\
l^{\prime} & s^{\prime} & j^{\prime}
\end{array}\right\}\left\{\begin{array}{ccc}
1 & 1 & k \\
\frac{1}{2} & \frac{1}{2} & \ddot{s} \\
\frac{1}{2} & \frac{1}{2} & s^{\prime}
\end{array}\right\} \\
& \times \sum_{a+b=2} \frac{p^{\prime a} \ddot{p}^{b}}{\sqrt{(2 a) !(2 b) !}} \sum_{\bar{l}} \hat{\bar{l}}_{\bar{l}}^{\rho N N}\left(p^{\prime}, \ddot{p}\right) \\
& \left.\times\left\{\begin{array}{lll}
a & b & 2 \\
\ddot{l} & l^{\prime} & \bar{l}
\end{array}\right\} C\left(a 0 \bar{l} 0, l^{\prime} 0\right) C(b 0 \bar{l} 0, \ddot{l} 0)\right] .
\end{aligned}
$$

Note that in the case of $\Lambda_{\pi N \Delta}=\Lambda_{\pi N N}$ (as was assumed in [6] and also used by us in the applications shown in this study), the function $H_{l}^{\pi N N-\pi N \Delta}\left(p^{\prime \prime \prime}, \dot{p}\right)$ is equal to $H_{l}^{\pi N N}\left(p^{\prime \prime \prime}, \dot{p}\right)$ defined in eq. (A.19) and the function $\tilde{H}_{l}^{\pi N N-\pi N \Delta}\left(p^{\prime \prime \prime}, \dot{p}\right)$ is equal to $\tilde{H}_{l}^{\pi N N}\left(p^{\prime \prime \prime}, \dot{p}\right)$ given by

$$
\begin{aligned}
& \tilde{H}_{\bar{l}}^{\pi N N}\left(p^{\prime \prime \prime}, \dot{p}\right)=-\frac{m_{\pi}^{2}}{p^{\prime \prime \prime} \dot{p}}\left(Q_{\bar{l}}\left(B_{m_{\pi}}\right)-Q_{\bar{l}}\left(B_{\Lambda_{\pi N N}}\right)\right) \\
& -\frac{\Lambda_{\pi N N}^{2}-m_{\pi}^{2}}{2\left(p^{\prime \prime \prime} \dot{p}\right)^{2}} \Lambda_{\pi N N}^{2} Q_{\bar{l}}^{\prime}\left(B_{\Lambda_{\pi N N}}\right) .
\end{aligned}
$$

Open Access This article is distributed under the terms of the Creative Commons Attribution Noncommercial License which permits any noncommercial use, distribution, and reproduction in any medium, provided the original author(s) and source are credited.

\section{References}

1. S.A. Coon, M.D. Scadron, P.C. Mcnamee, B.R. Barret, D.W.E. Blatt, B.H.J. McKellar, Nucl. Phys. A 317, 242 (1979).

2. R.G. Ellis, S.A. Coon, B.H.J. McKellar, Nucl. Phys. A 438, 631 (1985).

3. S.A. Coon, W. Glöckle, Phys. Rev. C 23, 1790 (1981).

4. S.A. Coon, M.T. Pena, Phys. Rev. C 48, 2559 (1993).

5. J.L. Friar, D. Hüber, U. van Kolck, Phys. Rev. C 59, 53 (1999).

6. A. Stadler, J. Adam jr., H. Henning, P.U. Sauer, Phys. Rev. C 51, 2896 (1995).

7. H. Witała, D. Hüber, W. Glöckle, J. Golak, A. Stadler, J. Adam jr., Phys. Rev. C 52, 1254 (1995).

8. H. Witała et al., Phys. Rev. C 63, 024007 (2001) and references therein.

9. J. Kuroś-Żołnierczuk et al., Phys. Rev. C 66, 024003 (2002).

10. J. Kuroś-Żołnierczuk et al., Phys. Rev. C 66, 024004 (2002).

11. J. Golak et al., Eur. Phys J. A 43, 241 (2010).

12. V. Bernard, E. Epelbaum, H. Krebs, Ulf-G. Meißner, Phys. Rev. C 77, 064004 (2008).

13. A. Nogga, D. Hüber, H. Kamada, W. Glöckle, Phys. Lett. B 409, 19 (1997).

14. D. Hüber, H. Kamada, H. Witała, W. Glöckle, Acta Phys. Pol. B 28, 1677 (1997).

15. W. Glöckle, H. Witała, D. Hüber, H. Kamada, J. Golak, Phys. Rep. 274, 107 (1996). 
16. W. Glöckle, The Quantum Mechanical Few-Body Problem (Springer-Verlag, Berlin-Heidelberg, 1983).

17. D. Hüber, H. Kamada, H. Witała, W. Glöckle, Few-Body Syst. 16, 165 (1994).

18. R. Lazauskas, Few-Body Syst. 46, 37 (2009).
19. Mathematica $^{(}$, Version 7.0 (Wolfram Research, Inc., Champaign, IL, 2008).

20. R. Balian, E. Berezin, Nuovo Cimento B 61, 403 (1969).

21. H. Witała et al., Phys. Lett. B 447, 216 (1999).

22. H. Witała et al., Phys. Rev. C 68, 034002 (2003). 